(C) 2006 International Press

Adv. Theor. Math. Phys. 10 (2006) 181-216

\title{
Spacetime foam in twistor string theory
}

\section{Sean A. Hartnoll ${ }^{1}$ and Giuseppe Policastro ${ }^{2}$}

${ }^{1}$ DAMTP, Centre for Mathematical Sciences, Cambridge University, Wilberforce Road, Cambridge CB3 OWA, UK

s.a.hartnoll@damtp.cam.ac.uk

${ }^{2}$ Ludwig-Maximilians-Universität, Theoretische Physik, Theresienstr. 37, 80333 München, Germany

policast@theorie.physik.uni-muenchen.de

\begin{abstract}
We show how a Kähler spacetime foam in four dimensional conformal (super)gravity may be mapped to twistor spaces carrying the D1 brane charge of the B model topological string theory. The spacetime foam is obtained by blowing up an arbitrary number of points in $\mathbb{C}^{2}$ and can be interpreted as a sum over gravitational instantons. Some twistor spaces for blowups of $\mathbb{C}^{2}$ are known explicitly. In these cases, we write down a meromorphic volume form and suggest a relation to a holomorphic superform on a corresponding super Calabi-Yau manifold.
\end{abstract}

e-print archive: http://lanl.arXiv.org/abs/hep-th/0412044 


\section{CONTENTS}

1. Introduction

2. Twistor correspondence and super Calabi-Yau manifolds

2.1. Twistor spaces

2.2. Twistorial super Calabi-Yau manifolds

3. Twistor spaces of Kähler manifolds and D1 brane charge

4. Twistor spaces for blowups

4.1. Complex geometry and topology of the twistor spaces

4.2. The meromorphic volume form 197

4.3. A holomorphic superform 199

4.4. Integrating the 3-form 202

5. Conclusions and discussion 205

$\begin{array}{ll}\text { Acknowledgments } & 207\end{array}$

$\begin{array}{lll}\text { Appendix A. } & \text { Jet bundles } & 207\end{array}$

$\begin{array}{lll}\text { Appendix B. Calculation of } b_{3}(\widetilde{Z}) & 209\end{array}$

Appendix C. Geometric transitions and topology of the twistor space 209

C.1. Calculation of $b_{2}\left(\widetilde{Z}_{\text {def. }}\right) \quad 211$

C.2. Betti numbers of the resolved space 212

Appendix D. Small resolution versus blowup 213

$\begin{array}{ll}\text { References } & 214\end{array}$ 


\section{Introduction}

The fate of spacetime at small length scales remains an outstanding problem in gravitational physics. The expectation of severe fluctuations in geometry and topology at the Planck length has lead to a picture of spacetime foam $[1,2]$. However, it is difficult to reliably quantify Planck scale physics using general relativity or superstring theory. In particular, the semiclassical approximation which underpins much of our knowledge of quantum gravity is unlikely to be valid at such small scales.

It is therefore rather impressive that the full perturbative partition function of Kähler gravity [3], a topological gravity theory in six dimensions, has recently been shown to be expressible as a sum over blowups of an asymptotically fixed toric Calabi-Yau manifold [4]. This explicit realization of spacetime foam was possible because Kähler gravity is the spacetime theory of topological A model string theory [5]. The topological A model on toric Calabi-Yau manifolds is under good computational control $[6,7]$.

Combining the A model picture with two other recent developments in topological string theory suggests an approach to spacetime foam in a four dimensional theory of gravity. First, it has been conjectured that $\mathcal{N}=4$ conformal supergravity in four dimensions has a dual description as the topological B model string theory on $\mathbb{C P}^{3 \mid 4}[8,9]$. These beautiful works have combined the richness of string theory with the mathematical structure of twistor theory $[10,11]$. In conformal gravities and supergravities, the Einstein-Hilbert action is replaced by

$$
S_{\mathrm{CG}} \propto \int d \Omega C_{a b c d} C^{a b c d}
$$

where $C_{a b c d}$ is the Weyl tensor. This action is formally similar to the YangMills action. Like the Yang-Mills action, it is conformally invariant and power-counting renormalizable. Despite these attractive features, such theories are generally not thought to provide adequate theories of gravity because the action $S_{\mathrm{CG}}$ has a four-derivative kinetic term and consequently suffers from unitarity problems [12]. In a Euclidean context, this is manifested as the inner product not being positive definite. However, a duality with topological string theory may help to resolve this issue or at least understand it better.

Secondly, it has been conjectured that there exists an $S$-duality in topological string theory that relates the A model and B model on a given CalabiYau manifold $[13,14]$. The spacetime foam we mentioned above is due to the worldsheet instantons of the A model. Worldsheet instantons are $S$-dual to D1 branes, so one therefore expects that the dual B model spacetime foam 
will be described as a gas of D1 branes. In the context of twistor string theory, a D1 brane wraps a $\mathbb{C P}^{1}$ in $\mathbb{C P}^{3 \mid 4}$. Under the twistor correspondence, each $\mathbb{C P}^{1}$ in $\mathbb{C P}^{3 \mid 4}$ corresponds to a point in $\mathbb{R}^{4}=\mathbb{C}^{2}$. A simple operation that can be performed at a given point is a blowup, that is, replacing the point with a finite $\mathbb{C P}^{1}$. This leads to the suggestion [13] that spacetime foam in conformal supergravity is described as a sum over blowups, with a twistorial description given as a gas of D1 branes in the topological B model. This idea is consistent with the fact that [9] the perturbative states of the B model are given by the complex structure deformations of the target space. When the target space is a twistor space, these deformations are mapped to fluctuations of the metric, i.e., perturbative states of gravity. Considering D1 branes then means extending this correspondence to a nonperturbative sector. The objective of this work is to start to flesh out this correspondence.

There is a further mathematical fact that supports the notion of spacetime foam in conformal gravity as a sum over blowups of $\mathbb{C}^{2}$. This comes from considering gravitational instantons in conformal gravity. Again like the Yang-Mills action, the action $S_{\mathrm{CG}}$ is minimised by self-dual or anti-self-dual instantons with

$$
C_{a b c d}= \pm \frac{1}{2} \varepsilon_{a b}{ }^{e f} C_{e f c d} .
$$

A metric with a Weyl tensor satisfying (1.2) is called conformally (anti)-selfdual. A manifold is called conformally (anti)-self-dual if it admits such a metric. The twistor correspondence $[10,11]$ states that there is a one-to-one correspondence between conformally (anti)-self-dual manifolds and a certain class of three complex-dimensional manifolds with a real structure.

Given a suitable normalization, the action evaluated for a gravitational instanton $M$ is a topological charge. The charge is the Hirzebruch signature of the four dimensional manifold

$$
S_{\mathrm{CG}}[M] \propto \frac{1}{96 \pi^{2}} \int d \Omega \varepsilon^{a b e f} C_{a b c d} C_{e f}^{c d}=\tau=b_{2}^{+}-b_{2}^{-},
$$

where $b_{2}^{ \pm}$are the second Betti numbers of the manifold.

Blowing up a point of a four-manifold $M$ is topologically equivalent to performing a connected sum with $\overline{\mathbb{C P}^{2}}$. Partially because of this fact, and following much of the mathematical literature, we shall work with anti-selfdual rather than self-dual manifolds. This choice is related to a choice of orientation. ${ }^{1}$ Thus we write

$$
M \mapsto M^{\prime}=M \# \overline{\mathbb{C P}^{2}}
$$

\footnotetext{
${ }^{1} \mathbb{C P}^{2}$ and $\overline{\mathbb{C P}^{2}}$ are trivially homeomorphic, but the homeomorphism is not orientation preserving.
} 
We then have that performing the connected sum with $\overline{\mathbb{C P}^{2}}$ decreases the signature by one

$$
\tau\left(M^{\prime}\right)=\tau(M)-1 .
$$

A particular case of this is $M^{\prime}=\mathbb{C}^{2} \# \overline{\mathbb{C P}^{2}}$ which has $\tau\left(M^{\prime}\right)=-1$. Furthermore this $M^{\prime}$ is a conformally anti-self-dual manifold and therefore could be thought of as a "minimal" gravitational instanton. In general $M^{\prime}$ is not conformally anti-self-dual. There are however various deep results in the theory of anti-self-dual manifolds concerning direct sums with $\overline{\mathbb{C P}^{2}}[15-18]$. Amongst these is the statement that

$$
M=\mathbb{C}^{2} \# \overline{\mathbb{C P}^{2}} \overbrace{\# \cdots \#}^{n} \overline{\mathbb{C P}^{2}}
$$

is conformally anti-self-dual for any $n$. These particular manifolds have various technically pleasant properties. With some assumptions about the symmetry of the configurations of points of $\mathbb{C}^{2}$ which are blown up, explicit asymptotically flat anti-self-dual metrics are known, together with their twistor spaces $[19,20]$. These twistor spaces turn out to be bimeromorphic to projective varieties $[19,21]$ and the anti-self-dual metrics turn out to be Kähler [20].

The upshot of these mathematical remarks is that the sum over blowups can be thought of as a gas of $\mathbb{C}^{2} \# \overline{\mathbb{C P}^{2}}$ gravitational instantons. We thus reach the appealing picture that an instanton gas in conformal supergravity could correspond to the D1 brane gas in twistor string theory.

The idea of the vacuum of conformal gravity as a gas of gravitation instantons was explored in [22] following observations in [23]. That work considered the physics of instantons other than blowups of $\mathbb{C}^{2}$. Specifically, the paper studied the instantons $K 3$ and $T^{4}$, as well as the manifold $S^{2} \times S^{2}$ which solves the Euclidean equations of motion, although it is not conformally anti-self-dual and therefore not a minimum of the action. These other instantons should also have an interpretation in twistor string theory that would be interesting to understand.

In Section 2, we review the twistor correspondence between conformally anti-self-dual four-manifolds and complex 3-folds. We also review an argument of LeBrun that constructs a natural super Calabi-Yau manifold from a twistor space and comment on the topology of twistor spaces.

In Section 3, we recall some properties of the twistor spaces of Kähler antiself-dual manifolds. We use these properties to show that Kähler blowups in four dimensions are mapped via the Penrose transform to a D1 brane charge in twistor space. 
In Section 4, we recall an explicit construction of twistor spaces for $\mathbb{C} \# \overline{\mathbb{C P}^{2}} \# \cdots \# \overline{\mathbb{C P}^{2}}$, also due to LeBrun. We discuss various aspects of the geometry and topology of these spaces. Following the prescription developed in Section 3, we write down a meromorphic 3-form that may be integrated to give a D1 brane charge. The form is singular because the canonical bundle of the twistor space is nontrivial. We go on to consider a super Calabi-Yau extension of the twistor spaces and write down a global (3|4)-form. By integrating out the fermionic directions, we recover the meromorphic form on the manifold.

Section 5 contains our conclusions and a discussion. The appendices contain technical details of some of the computations done in the text, as well as a discussion of geometric transitions in the twistor space.

\section{Twistor correspondence and super Calabi-Yau manifolds}

\section{$2.1 \quad$ Twistor spaces}

Let us briefly review Penrose's construction of a complex three-manifold $(Z, J)$ from a four dimensional conformally anti-self-dual Riemannian manifold $(M,[g])[10,11]$. The manifold $Z$ is called the twistor space of $M$.

The Hodge ${ }^{*}$ operation acting on 2 -forms on $M$ defines a linear map $\Lambda^{2} T^{*} M \rightarrow \Lambda^{2} T^{*} M$ such that $*^{2}=1$. The eigenspaces corresponding to eigenvalues \pm 1 are the self-dual and anti-self-dual 2-forms on $M$, respectively. The consequent split $\Lambda^{2}=\Lambda^{+} \oplus \Lambda^{-}$corresponds to the factorization of the rotation group on the tangent space $S O(4) \cong S O(3) \times S O(3)$. We therefore have globally defined vector bundles $\Lambda^{ \pm}$of rank 3 with structure group $S O(3)$. The Riemannian metric on $M$ induces a metric on the fibres, so we can consider the bundle having the unit sphere in $\Lambda^{+}$as fibre. The total space of this $S^{2}$ bundle is the twistor space $Z=S\left(\Lambda^{+}\right)$as a Riemannian six-manifold.

It is convenient to have a different interpretation for the fibres. A normalised self-dual 2-form can be identified, using the metric, with an endomorphism $J: T M \rightarrow T M$ that is skew, $J^{*}=-J$, and further satisfies $J^{2}=-1$. A point $z \in Z$ on the fibre of $x \in M$ is then an almost complex structure $J_{x}$ on $T_{x} M$. Thus the $S^{2}$ bundle of self-dual 2-forms is also the bundle of complex structures over $M$. The tangent space of $Z$ splits into $T Z=T F \oplus T M$, where $F$ is the fibre. We can put an almost complex structure $J$ on $T_{z} Z$ by firstly defining it to be $J_{x}$ on $T_{x} M$ and then on the fibre taking the unique 
complex structure on $S^{2} \cong \mathbb{C P}^{1}$ (up to a sign, chosen to be compatible with the orientation). The central result is that this almost complex structure is integrable when the self-dual part of the Weyl tensor on $M$ vanishes, $C^{+}=0$. The twistor space of an anti-self-dual manifold is therefore a complex 3-fold. This statement and its converse is given in the following theorem.

Theorem $2.1[10,11]$. The almost complex twistor space $(Z, J)$ of $(M,[g])$ is a complex 3-manifold if and only if $C^{+}=0$. Conversely, a complex three-manifold arises by this construction if and only if it admits an antiholomorphic involution $\sigma: Z \rightarrow Z$ without fixed points and a foliation by $\sigma$-invariant rational curves $\mathbb{C P}^{1}$, each of which has normal bundle $\mathcal{O}(1) \oplus$ $\mathcal{O}(1)$.

The $\sigma$-invariant curves in the twistor space $Z$, called the real curves, correspond to points in the four-manifold $M$. Two simple examples of twistor spaces are as follows. The twistor space of $S^{4}$ with the conformal equivalence class of the round metric is $\mathbb{C P}^{3}$. The twistor space of $\overline{\mathbb{C P}^{2}}$ with the Fubini-Study metric is a flag manifold that may be described as the hypersurface $v \cdot w=0$ with $v, w \in \mathbb{C}^{3}$ homogeneous coordinates on $\mathbb{C P}^{2} \times \mathbb{C P}^{2}$.

The twistor space of $\mathbb{R}^{4}=\mathbb{C}^{2}$ is obtained by removing a rational curve from $\mathbb{C P}^{3}$, corresponding to removing a point from $S^{4}$ and conformally decompactifying. The resulting space is written $\mathbb{C P}^{\prime 3}$. We will not always indicate the removal of the rational curve explicitly.

The construction of $Z$ gives enough information to compute its Betti numbers using theorems on sphere bundles ([24], II, $\S 11)$. By definition, $Z$ is an orientable $S^{2}$ bundle over $M$, with associated vector bundle $\Lambda^{+}$ over $M$. On an $S^{n}$-bundle, there is always a global "angular" $n$-form $\psi$ whose restriction to each fibre generates the cohomology of the fibre. The form $\psi$ is not in general closed, the obstruction being given by the Euler class $e\left(\Lambda^{+}\right)$(see for example [25] for an introduction to characteristic classes). $\Lambda^{+}$ has rank 3 and therefore has an orientation-reversing automorphism, given by $x \rightarrow-x$ on the fibres, under which the Euler class changes sign. The Euler class must therefore vanish. The angular form is then a cohomology class that generates the cohomology of the fibres. The existence of such a cohomology class for an orientable sphere bundle is precisely the condition under which the Leray-Hirsch theorem states that the cohomology of the bundle factorizes

$$
H^{*}(Z) \cong H^{*}(M) \times H^{*}\left(S^{2}\right) .
$$

It is then immediate to derive

$$
b_{2}(Z)=b_{2}(M)+1 \quad \text { and } \quad b_{3}(Z)=2 b_{1}(M) .
$$


In particular, for the manifolds, we are mainly interested in

$$
M=\mathbb{C}^{2} \# \overline{\mathbb{C P}^{2}} \overbrace{\# \cdots \#}^{n} \overline{\mathbb{C P}^{2}},
$$

we have $b_{2}(Z)=n+1$ and $b_{3}(Z)=0$.

We have defined $Z$ as the sphere bundle of unit self-dual 2-forms or as the bundle of complex structures. There is a third useful description: when the manifold $M$ is spin, there are well-defined spin bundles $\Sigma^{ \pm}$, which are rank 2 smooth complex bundles. We have the identifications $\Lambda^{1}=\Sigma^{+} \otimes \Sigma^{-}$and $\Lambda^{ \pm}=\Sigma^{ \pm} \otimes_{S} \Sigma^{ \pm}$, where $\otimes_{S}$ denotes symmetrized tensor product. In this case $Z$ can also be defined as $\mathbb{P}\left(\Sigma^{+}\right)$, the projectivization of the spin bundle. This description is useful locally even when the manifold $M$ is not spin.

\subsection{Twistorial super Calabi-Yau manifolds}

In the context of the B model topological string theory, one must make the twistor space into a super Calabi-Yau manifold in order for the string theory to be free of anomalies [8]. The B model needs a nonvanishing global holomorphic volume form. Such a form does not exist for twistor spaces because the canonical line bundle $K=\Omega^{3}$ is nontrivial. To overcome this problem in the case of $\mathbb{C P}^{3}[8]$, which has $K=\mathcal{O}(-4)$, one can consider a fermionic rank 4 complex vector bundle

$$
E=\mathcal{O}(1) \oplus \mathcal{O}(1) \oplus \mathcal{O}(1) \oplus \mathcal{O}(1),
$$

over the twistor space $\mathbb{C P}^{3}$. The total space of this bundle is in fact the super Calabi-Yau $\mathbb{C P}^{3 \mid 4}$. The super Calabi-Yau property follows from the fact that the "Berezinian line bundle"

$$
B=K \otimes \Lambda^{4} E,
$$

is trivial because $\Lambda^{4} E=\mathcal{O}(4)$. The Berezinian line bundle is the generalization of the canonical bundle to include the fermionic coordinates.

Lebrun [26] has presented a natural generalization of this construction to any twistor space. The construction has two prominent features: first, that given any twistor space $Z$, it produces a super Calabi-Yau manifold by appending a fermionic rank 4 vector bundle $E$. Secondly, when restricted to any real curve in $Z$, the bundle becomes $\mathcal{O}(1) \oplus \mathcal{O}(1) \oplus \mathcal{O}(1) \oplus \mathcal{O}(1)$ over

$\mathbb{C P}^{1}$. This second point will probably be important for the local degrees of freedom in four dimensions to remain those of $\mathcal{N}=4$ conformal supergravity [9]. We give a proof of the second point in Appendix A. 
First note that any twistor space is spin [27]. Therefore, the inverse square root of the canonical bundle, $K^{-1 / 2}$, is well defined. The rank 4 vector bundle will be given by the 1 -jets

$$
E=J^{1}\left(K^{-1 / 2}\right) \text {. }
$$

Loosely, $J^{1}(L)$ for some line bundle $L$ has as fibre at each point the equivalence classes of sections of $L$ determined by their value and first derivatives at that point. More precisely, there is a short exact sequence

$$
0 \longrightarrow \Omega^{1} \otimes K^{-1 / 2} \longrightarrow J^{1}\left(K^{-1 / 2}\right) \longrightarrow K^{-1 / 2} \longrightarrow 0 .
$$

This sequence does not split for twistor spaces. In Appendix A, we show how the short exact sequence gives the transition functions for the jet bundle. The only result we need is that this sequence implies that

$$
\Lambda^{4} E=K^{-1 / 2} \otimes \Lambda^{3}\left(\Omega^{1} \otimes K^{-1 / 2}\right) .
$$

We define the Berezinian line bundle $B$ as before in (2.5) but now with $E$ given by (2.6). However, from (2.8), we now have that

$$
\begin{aligned}
B & =K \otimes K^{-1 / 2} \otimes \Lambda^{3}\left(\Omega^{1} \otimes K^{-1 / 2}\right) \\
& =K \otimes K^{-1 / 2} \otimes K^{-3 / 2} \otimes K \\
& =1,
\end{aligned}
$$

so the bundle is trivial and the total space is super Calabi-Yau.

Although we shall not use the details of the above argument here, the possibility of a super Calabi-Yau construction for general twistor spaces is important for the duality between conformal gravity and B model topological strings to be tenable.

\section{Twistor spaces of Kähler manifolds and D1 brane charge}

The complex geometry of the twistor space $Z$ encodes all the information about the conformal geometry of the underlying four-manifold $M$. We consider in this section the additional structure enjoyed by the twistor space when $M$ is a Kähler manifold [20]. A two complex dimensional Kähler metric is anti-self-dual if and only if it is scalar flat [17]. The extra structure will be of importance to us shortly because spacetime foam with sufficient symmetry admits a Kähler structure that is compatible with anti-self-duality.

Notice first of all that $Z$, being a sphere bundle, does not come with a zero section. In general, there is thus no canonical way of embedding $M$ as a submanifold in $Z$. However, when there is a Kähler form, this provides precisely a nowhere vanishing section of $\Lambda^{+}(M)$, and thus a section of the 
twistor fibration $S\left(\Lambda^{+}\right)$. This section gives us a complex submanifold of $Z$ diffeomorphic to $M$. More precisely:

Theorem 3.1 [28]. Let $\pi: Z \rightarrow M$ be the twistor fibration of an anti-selfdual four-manifold $(M,[g])$. Suppose $Z$ contains a complex hypersurface $D$ that is the image of a section of $\pi$. Let $J$ be the complex structure on $M$ determined by $D$. Then there is a metric $h \in[g]$ which is Kähler with respect to $J$ if and only if the line bundle of the divisor $[D]+\sigma[D]$ is isomorphic to $K_{Z}^{-1 / 2}$.

We can recover the Kähler form from the twistor data in the following way. From the previous theorem, a section $s \in H^{0}\left(Z, K^{-1 / 2}\right)$ has a simple zero on $D \cup \bar{D}$, with $\bar{D}=\sigma D$. Taking a cover of $Z$ given by $\left\{Z_{1}=Z \backslash D, Z_{2}=\right.$ $Z \backslash \bar{D}\}, s$ is holomorphic and nonzero on $Z_{1} \cap Z_{2}$ and so $r_{12}=s^{-2}$ defines a cohomology class $r \in H^{1}(Z, K)$. We now recall how the Penrose transform $[8,20]$ maps $r$ to a self-dual closed 2-form of type $(1,1)$ for the complex structure defined by $D$. The Penrose transform involves a contour integral along the twistor lines $F_{x} \cong \mathbb{C P}^{1}$, which are the fibres of the twistor fibration above each point $x \in M$. Let $\lambda^{a}$ be homogeneous coordinates on the $\mathbb{C P}^{1}$ fibre viewed as $\mathbb{P}\left(\Sigma^{+}\right)$and use standard spinor indices so that $v_{a} \in \Sigma^{+}, v_{\dot{a}} \in$ $\Sigma^{-}$. The spinor indices are raised and lowered with $\epsilon_{a b}$ and $\epsilon_{\dot{a} \dot{b}}$. A 1-form on $M$, for example, is written as $\phi_{a \dot{a}}$. Since $\left.K\right|_{F}=\mathcal{O}(-4)$, then $s_{x}=\left.s\right|_{F_{x}} \in$ $H^{0}\left(F_{x}, \mathcal{O}(2)\right)$, and one can write $s_{x}=\omega_{a b}(x) \lambda^{a} \lambda^{b}$. Notice therefore that $r_{x} \in H^{1}\left(F_{x}, \mathcal{O}(-4)\right)$. The Penrose transform associates to $r_{x}$ a self-dual 2 -form via a contour integral on $F_{x}$ :

$$
k_{a b}(x)=\oint_{\Gamma \subset F_{x}} \lambda_{c} d \lambda^{c} \lambda_{a} \lambda_{b} r_{x}=\oint_{\Gamma \subset F_{x}} \frac{\lambda_{c} d \lambda^{c} \lambda_{a} \lambda_{b}}{\left(\omega_{e f}(x) \lambda^{e} \lambda^{f}\right)^{2}}=\frac{\omega_{a b}(x)}{(\operatorname{det} \omega(x))^{3 / 2}} .
$$

It can be proven that $k$ is the Kähler form of a scalar-flat anti-self-dual metric on $M[20]$.

To see that this form is of type $(1,1)$ on $M$, notice [11] that for each point $\lambda \in F_{x}$, there is an isomorphism $T_{x}^{*} M \simeq \Sigma^{-}$given by $v^{a \dot{a}} \mapsto \phi^{\dot{a}}=\lambda_{a} v^{a \dot{a}}$. Because $\Sigma^{-}$is a complex vector space, this map induces a complex structure on $T^{*} M$ that depends on $\lambda$ up to multiplication by a number. With this complex structure, the 1 -forms $e^{\dot{a}}=\lambda_{b} d x^{b \dot{a}}$ are by definition of type $(1,0)$. We can decompose a 1 -form in its components of type $(1,0)$ and $(0,1)$ using the projectors $\Pi^{b}{ }_{a}=i \bar{\lambda}^{b} \lambda_{a}, \bar{\Pi}^{b}{ }_{a}=i \lambda^{b} \bar{\lambda}_{a}$, assuming $\lambda$ is normalized so that $\lambda^{a} \bar{\lambda}_{a}=-i$. Then we have $d x^{a \dot{a}}=\bar{\lambda}^{a} e^{\dot{a}}+\lambda^{a} \bar{e}^{\dot{a}}$. Inserting this relation into the expression (3.1) for the Kähler form, $k \propto \omega_{a b} d x^{a \dot{a}} d x^{b \dot{b}} \epsilon_{\dot{a} \dot{b}}$, we see that the terms of type $(2,0)$ and $(0,2)$ are proportional to $\omega_{a b} \lambda^{a} \lambda^{b}$. These terms vanish exactly at $\left\{s_{x}=0\right\}=(D \cup \bar{D}) \cap F_{x}$. However, this is precisely where 
the residue of the contour integral (3.1) lies and hence where the form is evaluated. It follows that the Kähler form is of type $(1,1)$ in the complex structure determined by $D$ (or $\bar{D}$ ), as it should be.

The main observation we make is that we can now write a natural meromorphic 3-form on $Z$ corresponding to $s^{-2}$. Locally, in a patch where the twistor fibration can be trivialized, it is given by

$$
\Omega=s^{-2} \lambda^{c} d \lambda_{c} \lambda_{a} \lambda_{b} d x^{a \dot{a}} d x^{b \dot{b}} \epsilon_{\dot{a} \dot{b}} .
$$

This 3 -form will have quadratic singularities on a $D \cup \bar{D}$. The previous discussion then shows that integrating along a contour in a fibre gives the Kähler form,

$$
\oint_{\Gamma \subset F_{x}} \Omega=k(x)
$$

It follows that we can recover the Kähler moduli of the four-manifold by integrating $\Omega$ on suitably chosen 3 -cycles in $Z$

$$
\int_{\Sigma^{(2)}} k=\int_{\Sigma^{(3)}} \Omega
$$

This relationship is exciting because it relates a measure of four dimensional spacetime foam to an integral in six dimensions that detects D1 brane charge. Such a relationship is what we had anticipated in the introduction. It might seem contradictory that in $(3.3)$, a $(1,1)$-form in four dimensions is related through a contour integral to a $(3,0)$-form in six dimensions. However, this is what happens as we will now illustrate for the case of $\mathbb{C P}^{3}$.

In Section 4, we will explicitly construct the meromorphic 3-form for a class of twistor spaces which are bimeromorphically algebraic. Let us now, as an example, spell out this construction for the case of $\mathbb{C P}^{3}$ (strictly $\mathbb{C P}^{\prime 3}$ ). The corresponding four-manifold is just $\mathbb{C}^{2}$. The divisors $D$ and $\bar{D}$ will intersect on a real line which is the line that should be removed from $\mathbb{C P}^{3}$ to get the twistor space for $\mathbb{C}^{2}$ rather than $S^{4}$. In fact, divisors on $\mathbb{C P}^{3}$ are hyperplane sections and they always intersect at a line. We use here the notation of [8] so that $\mathbb{C P}^{3}$ has homogeneous coordinates $\left\{\mu_{\dot{a}}, \lambda^{a}\right\}$. The fibre over a point $x$ of $\mathbb{C}^{2}$ is then

$$
\mu_{\dot{a}}=x_{a \dot{a}} \lambda^{a},
$$

and removing the point at infinity means that $\lambda^{a} \neq(0,0)$. It is easy to see that the 3 -form (3.2) becomes

$$
\Omega=s^{-2} \lambda^{a} d \lambda_{a} \wedge d \mu^{\dot{a}} \wedge d \mu_{\dot{a}} .
$$

Without the prefactor $s^{-2}$, this is the natural 3 -form on $\mathbb{C P}^{3}$ with values in $\mathcal{O}(4)$. The prefactor turns it into an honest (scale-invariant) 3 -form but with singularities. Another way to obtain a well-defined form is to add 
fermionic directions [8]. We shall discuss the relationship between these singular forms and the superforms later. The meaning of the singularity has been discussed above; for each choice of a section $s$, we get a Kähler form on $\mathbb{C}^{2}$. The involution acts as $\sigma: \lambda^{a} \mapsto \bar{\lambda}_{a}$. An example of a section we could take is $s=\delta_{a b} \lambda^{a} \lambda^{b}$. We see that $D=\left\{\lambda^{1}=i \lambda^{2}\right\}, \bar{D}=\left\{\lambda^{1}=-i \lambda^{2}\right\}$ and $D \cap \bar{D}=\left\{\lambda^{a}=0\right\}$ which is the line at infinity that we removed in these coordinates. The Kähler form computed from $s$ is then

$$
k=\delta_{a b} \epsilon_{\dot{a} \dot{b}} d x^{a \dot{a}} \wedge d x^{b \dot{b}}=d x^{11} \wedge d x^{12}+d x^{21} \wedge d x^{22}=e^{\dot{1}} \wedge \bar{e}^{\dot{2}}-e^{\dot{2}} \wedge \bar{e}^{\dot{1}},
$$

where we used the normalization $\lambda^{a} \bar{\lambda}_{a}=-i$ and defined $e^{\dot{a}}=\lambda_{b} d x^{b \dot{a}}$, as discussed above. We see that (3.7) is indeed a $(1,1)$-form and gives a selfdual Kähler form on $\mathbb{C}^{2}$. Of course, $\mathbb{C}^{2}$ does not have nontrivial 2-cycles about which we can integrate the Kähler form. This corresponds to the absence of D1 brane charge in $\mathbb{C P}^{3}$.

It is remarkable that the twistor correspondence allows us to associate a meromorphic form in three complex dimensions to an anti-self-dual Kähler manifold $M$ in four real dimensions. This appears to set up a correspondence between a physical theory on $M$ that depends on the Kähler moduli and a theory on $Z$ that depends on the complex moduli. This is reminiscent of mirror symmetry, but with a change in dimensions involved. On the complex side of the story, we are embedding the theory into the topological B model on a supermanifold extension of $Z$ while on the Kähler side, we are embedding the theory into (super)conformal gravity.

The fact that we would like to identify the integral of the $(3,0)$-form around a 3-cycle as D1 brane charge is the statement that [29] in the presence of D1 branes, or in a space obtained from the backreaction of D1 branes, we have

$$
\int_{\Sigma^{(3)}} \Omega=g_{s} N
$$

where $N$ is the number of D1 branes linked by $\Sigma^{(3)}$. In the topological string context $N$ is of course an integer. Comparing with (3.4) then suggests a tantalizing quantization of the Kähler moduli in the four dimensional theory. This seems very similar to the quantization of the Kähler moduli that was found in the six dimensional spacetime foam studied in [4].

We noted below equation (2.3) that for the twistor spaces that we are interested in, describing spacetime foam, we have $b_{3}(Z)=0$. Thus the only way for the closed 3 -form $\Omega$ to admit a nonzero period is if it is singular. Such a singularity is related to the nontriviality of the canonical bundle. More concretely, we have that $d \Omega$ will be a 4 -form with delta function support on the divisor $D \cup \bar{D}$. One might have expected support on a 2-cycle rather 
than a 4-cycle, corresponding to the location of a brane. This is not possible however as the singular locus of a meromorphic 3-form will always be a complex codimension-one submanifold. Thus, we should think of the twistor space as already incorporating the backreaction of the D1 branes.

In the following section, we shall study explicitly known twistor spaces for $\mathbb{C}^{2}$ blown up at $n$ points using the framework we have just described. Explicit asymptotically flat anti-self-dual Kähler metrics and the corresponding twistor spaces are known in the case when the $n$ blown-up points are collinear [19]. They are also known to exist if the configuration of points is sufficiently close to collinear [20]. Therefore, the spacetime foam in conformal (super)gravity has a "Kähler sector" and it is this sector which we are studying.

\section{Twistor spaces for blowups}

\subsection{Complex geometry and topology of the twistor spaces}

Lebrun has explicitly constructed twistor spaces for Kähler anti-self-dual metrics on $M=\mathbb{C}^{2} \# \overline{\mathbb{C P}^{2}} \# \cdots \# \overline{\mathbb{C P}^{2}}[19,20]$. The spaces are not the twistor spaces of the most general conformally anti-self-dual metric on $M$ but are rather special points in the moduli space of anti-self-dual metrics at which all the blown-up points are collinear. These have the remarkable property that the corresponding twistor spaces are bimeromorphically algebraic. That is, we may start with a singular algebraic 3 -fold and obtain the twistor space by performing blowups and blowdowns. These spaces will allow a concrete realization of the ideas discussed in previous sections and will further allow us to make a connection with super Calabi-Yau manifolds.

The construction starts by considering a singular 3 -fold $\widetilde{Z}$. Take $\mathbb{C P}^{1} \times$ $\mathbb{C P}^{1}$ with homogeneous coordinates $\left[z_{0}, z_{1}\right]$ and $\left[\zeta_{0}, \zeta_{1}\right]$. One then considers the total space of a projectivized bundle over $\mathbb{C P}^{1} \times \mathbb{C P}^{1}$

$$
\mathcal{B} \equiv \mathbb{P}[\mathcal{O}(n-1,1) \oplus \mathcal{O}(1, n-1) \oplus \mathcal{O}],
$$

with coordinates for the fibres $x \in \mathcal{O}(n-1,1), y \in \mathcal{O}(1, n-1)$ and $t \in \mathcal{O}$. Note that $\mathcal{B}$ is a four complex dimensional manifold obtained from $\mathbb{C}^{7}$ via the following three identifications

$$
\begin{aligned}
{\left[z_{0}, z_{1}\right] } & \sim \lambda\left[z_{0}, z_{1}\right], \\
{\left[\zeta_{0}, \zeta_{1}\right] } & \sim \mu\left[\zeta_{0}, \zeta_{1}\right], \\
{[x, y, t] } & \sim \nu\left[\lambda^{n-1} \mu x, \lambda \mu^{n-1} y, t\right] .
\end{aligned}
$$


Now consider the singular 3 -fold given by a hypersurface

$$
\widetilde{Z} \subset \mathcal{B}
$$

defined by

$$
F \equiv x y-t^{2} \prod_{j=1}^{n} P^{j}=0 .
$$

In this expression, the $P^{j}$ are $n$ polynomials in $\left(z_{0}, z_{1}, \zeta_{0}, \zeta_{1}\right)$ which we now define. Note that the manifold $\mathbb{C P}^{1} \times \mathbb{C P}^{1}$ admits an anti-holomorphic involution $\sigma$ given by

$$
\sigma\left(\left[z_{0}, z_{1}\right],\left[\zeta_{0}, \zeta_{1}\right]\right) \longmapsto\left(\left[\bar{\zeta}_{0}, \bar{\zeta}_{1}\right],\left[\overline{z_{0}}, \overline{z_{1}}\right]\right) .
$$

In order to obtain later the twistor space for the noncompact manifold $M$, we should remove from $\mathbb{C P}^{1} \times \mathbb{C P}^{1}$ the line $S \subset \mathbb{C P}^{1} \times \mathbb{C P}^{1}$ given by the fixed points of $\sigma$. This will correspond to removing the point at infinity in four dimensions. Consider $n \sigma$-invariant curves in $\mathbb{C P}^{1} \times \mathbb{C P}^{1}-S$, which we denote $\left\{C^{i}\right\}_{i=1}^{n}$. These curves are specified as the zeros of $n$ polynomials

$$
P^{i} \in \Gamma\left(\mathbb{C P}^{1} \times \mathbb{C P}^{1}, \mathcal{O}(1,1)\right) .
$$

Explicitly these are

$$
P^{i}=a_{m n}^{i} \zeta_{m} z_{n}, \text { with } a^{i}=a^{i \dagger} .
$$

We assume that the curves are nondegenerate and generic, implying that they all mutually intersect at precisely two points. The curves are illustrated in figure 1.

The 3-fold $\widetilde{Z}$ is described topologically as follows. Away from the curves $P^{i}=0$ in $\mathbb{C P}^{1} \times \mathbb{C P}^{1}$, the manifold is a $\mathbb{C P}^{1}$ fibration over $\mathbb{C P}^{1} \times \mathbb{C P}^{1}$. Above the union of the curves $X^{n}=\cup_{i=1}^{n} C^{i}$, the fibration degenerates to two spheres joined at a point, which may be written $S^{2} \vee S^{2}$. The degeneration is regular except for the points where two curves intersect. Each pair

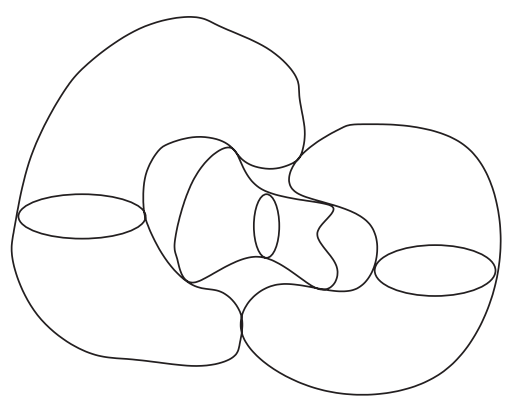

Figure 1: 2-cycles $C^{1}, C^{2}$ and $C^{3}$ mutually intersecting at two points. 
of curves intersect at two points, so there will be a total of $n(n-1)$ singular points. The fibration is shown in figure 2 .

We can make a simple observation on the topology of this singular space. Note that $S^{2} \vee S^{2}$ is formed by collapsing an $S^{1}$ in $\mathbb{C P}^{1}=S^{2}$. Therefore, every closed loop in $X^{n}$ bounds a disc, $D^{2}$, with an $S^{1}$ fibration that collapses on the boundary of the disc. This gives a homology $S^{3}$ in the full space. So we have

$$
b_{3}(\widetilde{Z})=b_{1}\left(X^{n}\right) .
$$

It is easy to calculate $b_{1}\left(X^{n}\right)$ using the Mayer-Vietoris sequence. The computation is given in Appendix B. The result is

$$
b_{1}\left(X^{n}\right)=b_{3}(\widetilde{Z})=(n-1)^{2} .
$$

To obtain the twistor space, we must resolve the singularities of $\tilde{Z}$. This can be done in two ways, which give the same result: by a sequence of blowups and blowdowns or by taking small resolutions. In the text, we explain the second method, more familiar to physicists, and discuss the equivalence with the first method in Appendix D.

Under the assumption of genericity that we are making, the curves $C^{i}$ have only isolated intersections. We can then choose local coordinates $\left\{w_{1}, w_{2}\right\}$ for $\mathbb{C P}^{1} \times \mathbb{C P}^{1}$ centred at one of the intersections and such that the two curves are given by the equations $w_{1}=0, w_{2}=0$, respectively. The equation for the 3 -fold then locally reads $x y=w_{1} w_{2}$. This has a conifold singularity, and it is well known that it can be resolved in such a way that the singular point is replaced by a $\mathbb{C P}^{1}$. The resolved space is (locally) the total space of a bundle $\mathcal{O}(-1) \oplus \mathcal{O}(-1) \rightarrow \mathbb{C P}^{1}$. If this bundle has coordinates $\left\{u, v,\left[Z_{1}, Z_{2}\right]\right\}$, one defines a map

$$
\left(x, y, w_{1}, w_{2}\right)=\left(u Z_{1}, v Z_{2}, u Z_{2}, v Z_{1}\right) .
$$

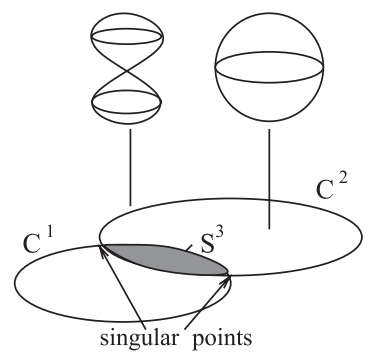

Figure 2: Two curves, $C^{1}$ and $C^{2}$, in $\mathbb{C P}^{1} \times \mathbb{C P}^{1}$ with their singular fibration. The shaded region in the base corresponds to an $S^{3}$ in the full space. 
The map is an isomorphism away from the zero section $u=v=0$ that is mapped to the singular point. The two intersecting curves are $C^{1}=\{x=$ $\left.y=w_{1}=0\right\}$ and $C^{2}=\left\{x=y=w_{2}=0\right\}$. In terms of the new coordinates, one has $C^{1}=\left\{u=Z_{2}=0\right\}, C^{2}=\left\{v=Z_{1}=0\right\}$ and therefore they have no intersection in the resolved space. Figure 3 shows the disconnection of the cycles after the small resolution.

The fact that the cycles $C^{i}$ above which the $S^{2}$ fibre degenerates are no longer connected implies that the resolved space satisfies

$$
b_{3}(Z)=0,
$$

in agreement with our observation below equation (2.3). In Appendix C, we give a more rigorous direct calculation of the Betti numbers of the twistor space to further find

$$
b_{2}(Z)=n+1
$$

again in agreement with the general considerations in Section 2.1.

One might wonder what happens if we deform the nodal singularities rather than taking small resolutions. These two desingularization are related through geometric transitions that are of general interest in string theory. The deformed space however is not a twistor space. In the course of calculating the Betti numbers of $Z$ in Appendix $\mathrm{C}$, we consider deformation of the singularities as well as small resolutions.

There is one final technical step in the construction of the twistor space. The singular space $\widetilde{Z}$ includes the divisors

$$
E_{x}=\{x=t=0\} \quad \text { and } \quad E_{y}=\{y=t=0\},
$$

that are not affected by the resolutions we have just described. Each of these may be identified via projection to the base with $\mathbb{C P}^{1} \times \mathbb{C P}^{1}$, and their

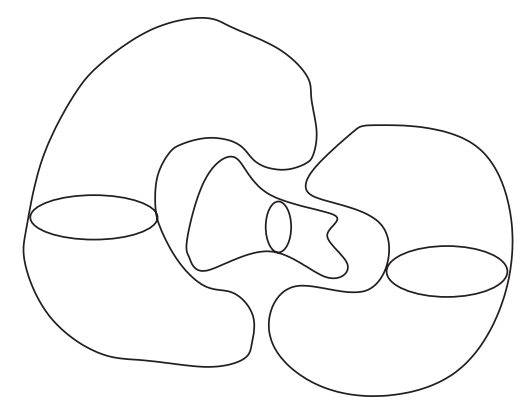

Figure 3: The resolution disconnects the cycles of figure 1. The figure is a little misleading as the separation is in the resolved $\mathbb{C P}^{1}$ rather than in the $\{z, \zeta\}$ coordinates. 
normal bundles are $\mathcal{O}(-1,1-n)$ and $\mathcal{O}(1-n,-1)$, respectively. They may therefore be blown down to give curves $\mathbb{C P}^{1}$ with normal bundle $\mathcal{O}(1-n) \oplus$ $\mathcal{O}(1-n)$. Performing these two blowdowns gives the desired twistor space $Z$ of $M$.

\subsection{The meromorphic volume form}

In this subsection, we will write down the explicit meromorphic 3-form for the twistor spaces $Z$ that is associated to the four dimensional Kähler form in (3.2). In order to do this, we have to recall first the structure of the twistor lines [19] and secondly the fundamental divisor $D$ in $Z$ [20]. We will work in terms of the coordinates $\left\{x, y, t, z_{0}, z_{1}, \zeta_{0}, \zeta_{1}\right\}$ on $\mathbb{C}^{7}$ which are subject to the three $\mathbb{C}^{*}$ actions (4.2) and the hypersurface condition $F=0$ (4.4). Often we will work in a patch with, say, $z_{1} \neq 0$ and $\zeta_{1} \neq 0$. In this case, we will use coordinates $z=z_{0} / z_{1}$ and $\zeta=\zeta_{0} / \zeta_{1}$.

The generic twistor line is obtained by taking a real curve $C$ on $\mathbb{C P}^{1} \times$ $\mathbb{C P}^{1}$, distinct from all the curves $C^{i}$ that we considered previously. The defining equation for $C$ is a real polynomial of degree $(1,1)$

$$
H(z, \zeta)=b_{m n} \zeta_{m} z_{n} \quad \text { with } b=b^{\dagger} .
$$

Now, restriction of $P$ to $C$ is a real polynomial of degree $2 n$ with zeros at the points where $C$ intersects the $C^{i}$ s. We can write $\left.P\right|_{C}=f \sigma^{*}(f)$ for some $f$ of degree $n$. It turns out that the twistor lines are given by [19]

$$
\left\{H(z, \zeta)=0, \quad x=t \mathrm{e}^{i \alpha} f, \quad y=t \mathrm{e}^{-i \alpha} \sigma^{*}(f)\right\},
$$

with $\alpha$ fixed for a given twistor line. If the curve $C$ happens to pass through an intersection $C^{i} \cap C^{j}$, we take its proper transform in the resolution of the singular space $\widetilde{Z}$. The (proper transforms of the) curves $C^{i}$ themselves are also twistor lines. The equations (4.15) describe a 4-real parameter family of curves with three parameters from $H$ and 1 from $\alpha$. There are also some other twistor lines that pass through $t=0$ and are given by $\{x, y, t=0$, $\zeta=\bar{z}\}$. Notice that these lines intersect the blown-down divisors $E_{x}$ and $E_{y}$ of (4.13).

The divisor $D$ turns out to be given as follows. Start with the divisor $\tilde{D}$ in $\tilde{Z}[20]$

$$
\tilde{D}=\{z=c\},
$$

in terms of the local coordinate $z$. Except for a finite number of values of $c, \tilde{D}$ will be disjoint from the singularities of $\tilde{Z}$ and therefore is unaffected by the resolution. Thus our spaces are almost foliated by these divisors. This last 
property is closely related to that of being bimeromorphically algebraic [30]. The divisor $D$ is the image of $\tilde{D}$ under the projection

$$
b: \tilde{Z} \longrightarrow Z \text {, }
$$

given by the blowdowns of the divisors $E_{x}$ and $E_{y}$ (4.13). To see the topology of $D$ [20], note that it inherits from $Z$ the $S^{2}$ fibration of figure 2 over the $\{z=c\}$ plane that degenerates over the $n$ points where $\{z=c\}$ intersects the curves $C^{i}$. The collapse of an $S^{1}$ in the fibration results in $D$ being given topologically as $\mathbb{C}^{2}$ blown up at $n$ points. Thus, $D$ is homeomorphic to $M$ as expected.

From the expression for $\tilde{D}$ and the action of $\sigma$ (4.5), we have that

$$
D \cup \bar{D}=b(\{z=c\} \cup\{\zeta=\bar{c}\}) .
$$

One can easily see now that $D$ is indeed a section of the twistor fibration. When $z$ is fixed, the equations for the twistor lines (4.15) fix all the other coordinates and hence $D$ intersects each twistor line at precisely one point.

Now let $s$ be a holomorphic section of $K_{Z}^{-1 / 2}$ with divisor $D \cup \bar{D}$ as in Section 3. We construct a meromorphic 3 -form $\Omega_{Z}$ by multiplying $s^{-2}$, which is a meromorphic section of $K_{Z}$, by a section of $\Omega_{Z}^{3} \otimes K_{Z}^{-1}$. The latter is manifestly a trivial line bundle and so it has a unique global section, up to a scale. In order to exhibit this form explicitly, it is more convenient to work in $\widetilde{Z}$ than $Z$ because we have global coordinates on $\widetilde{Z}$. We should bear in mind however that $\widetilde{Z}$ is not the true twistor space. Our strategy is to construct a meromorphic 3 -form $\Omega_{\tilde{Z}}$ such that

$$
\Omega_{\widetilde{Z}}=b^{*} \Omega_{Z},
$$

where as before $b: \widetilde{Z} \rightarrow Z$. With a slight abuse of notation, we write the section $s$ using coordinates on $\widetilde{Z}$

$$
s=\left(z_{0}-c z_{1}\right)\left(\zeta_{0}-\bar{c} \zeta_{1}\right) .
$$

We may take $z=z_{0} / z_{1}$ to be a coordinate on the twistor fibre, as $z$ is transverse to $D$. Then take $\zeta=\zeta_{0} / \zeta_{1}$ and $t$ as coordinates on $D$. We need a scale-invariant meromorphic 3 -form with quadratic poles along $\tilde{D} \cup \overline{\tilde{D}}$. An expression satisfying these requirements is

$$
\Omega_{\tilde{Z}}=\frac{d z \wedge d \zeta \wedge d t}{t(z-c)^{2}(\zeta-\bar{c})^{2}} .
$$

In the next section, we will explain how this form can be obtained using the method of Poincaré residue. This method will also give an expression for the form in other coordinate charts. It might appear worrysome that the form (4.21) has a singularity at $t=0$. In fact it is to be expected: if $\Omega_{Z}$ is to be singular on $D \cup \bar{D}$, its pull-back will be singular on $b^{-1}(D)=\tilde{D} \cup \overline{\tilde{D}} \cup\{t=0\}$. 
This can be seen explicitly by studying the neighbourhood of the blown-down divisor using a coordinate change similar to that used in Appendix D. Even without doing a computation, one can argue that a meromorphic 3 -form must have singularities along a divisor, i.e., a codimension 1 subvariety, but the locus $\{t=0\}$ is blown-down by $b$ and so is not a divisor in $Z$. Therefore, $\{t=0\}$ cannot be a pole of $\Omega_{Z}$.

\subsection{A holomorphic superform}

In this subsection, we make an informed guess at the holomorphic volume form on a super Calabi-Yau manifold constructed from the twistor spaces for $M=\mathbb{C}^{2} \# \overline{\mathbb{C P}^{2}} \# \cdots \# \overline{\mathbb{C P}^{2}}$ that we described in the previous subsections. This superform will have the property that when we integrate out the fermionic directions, we obtain the meromorphic volume form that we have just described.

The first step is to construct a holomorphic volume form $\Omega_{3 \mid 4}$ on a supermanifold extension of the singular space $\widetilde{Z}$. Recall that the bosonic part of this space was given as the hypersurface $F=0$ in the 4 -fold $\mathcal{B}(4.4)$. The supermanifold extension of $\widetilde{Z}$ will be given shortly as a hypersurface $\mathcal{F}=0$ in a supermanifold extension of $\mathcal{B}$. From now on, we shall use $Z_{S}, \widetilde{Z}_{S}$ and $\mathcal{B}_{S}$ to refer to the supermanifold extensions of the respective manifolds.

There is a well-known way of constructing holomorphic volume forms on hypersurfaces from a singular holomorphic form in the ambient space, called the Poincaré residue map [31]. In our context, this map takes a section of $\Omega^{4 \mid 4}\left(\mathcal{B}_{S}, \mathcal{F}\right)$, that is $(4 \mid 4)$-forms on $\mathcal{B}_{S}$ with a simple pole along $\mathcal{F}=0$, to a section of $\Omega^{3 \mid 4}\left(\widetilde{Z}_{S}\right)$. Thus, we begin by writing down a section of $\Omega^{4 \mid 4}\left(\mathcal{B}_{S}, \mathcal{F}\right)$.

Recall that $\mathcal{B}$ is constructed from three $\mathbb{C}^{*}$ actions on $\mathbb{C}^{7}(4.2)$. We can work in terms of coordinates $\left\{z^{i}\right\}$ of $\mathbb{C}^{7}$. More concretely, in terms of the coordinates introduced earlier, we will have $\left\{z^{i}\right\}=\left\{x, y, t, z_{0}, z_{1}, \zeta_{0}, \zeta_{1}\right\}$. If we let $k$ denote a vector generating any of the three $\mathbb{C}^{*}$ actions, then a 4-form $\Omega_{\mathcal{B}}$ defined on $\mathcal{B}$ should only have legs pointing transversally to the orbits, that is $\iota_{k} \Omega_{\mathcal{B}}=0$. Further, the form must be a sum of terms that have the same overall scaling under the $\mathbb{C}^{*}$ actions (4.2). Starting from the holomorphic volume form on $\mathbb{C}^{7}$ :

$$
\Omega_{7}=d z^{1} \wedge \cdots \wedge d z^{7}
$$


and given that we also want a pole along $\mathcal{F}=0$, the natural form to write down is

$$
\Omega_{\mathcal{B}}=\frac{\iota_{k_{\lambda}} \iota_{k_{\mu}} \iota_{k_{\nu}} \Omega_{7}}{\mathcal{F}}
$$

In this expression, the various $\iota_{k}$ terms denote contraction with the generators of the three $\mathbb{C}^{*}$ actions (4.2). The numerator turns out to be the following 4-form:

$$
\iota_{k_{\lambda}} \iota_{k_{\mu}} \iota_{k_{\nu}} \Omega_{7}=(x d y d t-y d x d t+t d x d y)\left(\epsilon_{i j} z^{i} d z^{j}\right)\left(\epsilon_{k l} \zeta^{k} d \zeta^{l}\right) .
$$

However, the form (4.23) is not a 4 -form on $\mathcal{B}$ because it is not invariant under the rescalings $(4.2)$

$$
\Omega_{\mathcal{B}} \rightarrow \lambda^{2} \mu^{2} \nu \Omega_{\mathcal{B}}
$$

Therefore, in the spirit of [8], we will form a supermanifold $\mathcal{B}_{S}$ by adding a four dimensional fermionic vector bundle $E$ with local coordinates $d \eta^{1}, \ldots, d \eta^{4}$ to $\mathcal{B}$. The only property we require at this point is that the determinant line bundle $\Lambda^{4} E$ has the opposite scaling to (4.25). It follows that the form

$$
\Omega_{\mathcal{B}_{S}}=\Omega_{\mathcal{B}} d \eta^{1} d \eta^{2} d \eta^{3} d \eta^{4}
$$

is well defined on the supermanifold $\mathcal{B}_{S}$ and is a section of $\Omega^{4 \mid 4}\left(\mathcal{B}_{S}, \mathcal{F}\right)$.

The next step is to construct from $\Omega_{\mathcal{B}_{S}}$ a holomorphic volume form on the singular space $\widetilde{Z}_{S}$ that is given by $\mathcal{F}=0$ in $\mathcal{B}_{S}$ (4.4). The Poincaré residue map gives this form to be

$$
\Omega_{\widetilde{Z}_{S}}=\frac{\Omega_{\mathcal{B}_{S}}}{d \mathcal{F}}
$$

More precisely, one writes

$$
\Omega_{\mathcal{B}_{S}}=\frac{d \mathcal{F}}{\mathcal{F}} \wedge \eta
$$

and defines

$$
\left.\Omega_{\widetilde{Z}_{S}} \equiv \eta\right|_{\widetilde{Z}_{S}}
$$

It is clear that the residue is a well-defined form at all points where $d \mathcal{F} \neq 0$, but it can have singularities at $\mathcal{F}=d \mathcal{F}=0$. In the present context, the singularities will be at the singular points of $\tilde{Z}_{S}$.

At this point we need to discuss the expression for $\mathcal{F}$. The most nä̈ve thing we could do is to simply take $\mathcal{F}=F$, with $F$ given in (4.4). However, this turns out to be somewhat unsatisfactory. The reason we are trying to construct the holomorphic volume form is that we would like to integrate the form over a (3|4)-cycle in the supermanifold to obtain a D1 brane charge. This is the supermanifold generalization of (3.8). Using $\mathcal{F}=F$ in (4.27) would give a form whose only fermionic dependence is $d \eta^{1} d \eta^{2} d \eta^{3} d \eta^{4}$. However, upon Berezinian integration, this form will always integrate to zero. 
To get a nonzero answer, we need to integrate $\eta^{1} \eta^{2} \eta^{3} \eta^{4} d \eta^{1} d \eta^{2} d \eta^{3} d \eta^{4}$. The simplest way to obtain such a term and restrict to $F=0$ on the bosonic manifold is to take

$$
\mathcal{F} \equiv F+G \eta^{1} \eta^{2} \eta^{3} \eta^{4}
$$

Similar expressions arose in the hypersurfaces of supermanifolds considered by [32]. Note that if we do not do the fermionic integrations, then the D1 brane charge would not be a number but would be a section of a nontrivial bundle. This would then make it difficult to compare brane charge on different manifolds or to speak about the number of D1 branes.

In (4.30), $G$ must be such that the scaling of $G \eta^{1} \eta^{2} \eta^{3} \eta^{4}$ under the $\mathbb{C}^{*}$ actions is equal to the scaling of $F$. We further know from the definition of Berezinian integration that $\eta^{1} \eta^{2} \eta^{3} \eta^{4}$ must scale inversely to $d \eta^{1} d \eta^{2} d \eta^{3} d \eta^{4}$. Putting these facts together requires that $G$ scales as

$$
G \sim F d \eta^{1} d \eta^{2} d \eta^{3} d \eta^{4} \sim \frac{F^{2}}{d z^{1} \cdots d z^{7}}
$$

Guided by our findings of the previous subsection, note that a simple expression with the correct scaling is

$$
G=\frac{F}{t s^{2}}
$$

That is, we take the holomorphic volume form to be

$$
\Omega_{\widetilde{Z}_{S}}=\frac{\iota_{k_{\lambda}} \iota_{k_{\mu}} \iota_{k_{\nu}} \Omega_{7} d \eta^{1} d \eta^{2} d \eta^{3} d \eta^{4}}{d\left[F+\left(F / t s^{2}\right) \eta^{1} \eta^{2} \eta^{3} \eta^{4}\right]} .
$$

It is not clear to us that this construction is unique. However, it does appear to combine the objects that are given in a simple and natural way to obtain a well-defined form that, as we shall see shortly, trivializes the Berezinian line bundle. However, the proof of the pudding is in the eating and we shall see now that upon Berezinian integration this superform reduces to our previously obtained meromorphic volume form on $\widetilde{Z}$.

We work in the coordinate patch $z_{1} \neq 0, \zeta_{1} \neq 0$ and $y \neq 0$. Next, let us move $d x$ to the denominator in (4.33). This gives

$$
\Omega_{\widetilde{Z}_{S}} \propto \frac{z_{1} \zeta_{1} d z_{0} d \zeta_{0} d t d \eta^{1} d \eta^{2} d \eta^{3} d \eta^{4}}{1+\left(1 / t s^{2}\right) \eta^{1} \eta^{2} \eta^{3} \eta^{4}}
$$

The overall normalization is not fixed uniquely, so we will not keep track of it. If we expand the fermionic components of this superform, we find

$$
\Omega_{\widetilde{Z}_{S}}=z_{1} \zeta_{1} d z_{0} d \zeta_{0} d t d \eta^{1} d \eta^{2} d \eta^{3} d \eta^{4}+\Omega_{\widetilde{Z}} \eta^{1} \eta^{2} \eta^{3} \eta^{4} d \eta^{1} d \eta^{2} d \eta^{3} d \eta^{4}
$$


The first term in this expression is a global holomorphic superform, entirely analogous to that introduced in [8], that trivializes the Berezinian line bundle and makes the supermanifold a super Calabi-Yau manifold. The second term will recover the form $\Omega_{\widetilde{Z}}$ of (4.21) after Berezinian integration. One can also work in other coordinate patches, e.g. when $t \neq 0$, and obtain expressions similar to (4.21) but with $d t / t$ replaced by $d x / x$ or $d y / y$.

\subsection{Integrating the 3-form}

The form $\Omega_{\widetilde{Z}}$ we have constructed lives on the singular manifold $\widetilde{Z}$ and has poles at the singularities where $d F=0$. As we described in Section 4, the singularities of $\widetilde{Z}$ are removed by taking small resolutions (4.10). After the resolution, the poles in the 3 -form disappear. The easiest way to see this is to use local coordinates. The suitable coordinate change is that given in equation (4.10). As noted before, the singularities of $F=0$ are all locally of the conifold type $x y-w_{1} w_{2}=0$ and the 3 -form given by the residue map is locally

$$
\frac{d x d w_{1} d w_{2}}{x}
$$

when $x \neq 0$ and similarly in the other patches. After the resolution the form is given by

$$
d Z d u d v
$$

and so is manifestly smooth. Notice that a small resolution does not introduce exceptional divisors, so the canonical bundle does not change and the pull-back of the form will not have additional zeros or poles.

In this section, we will integrate $\Omega_{\widetilde{Z}}$ around a contour in the twistor lines as we described in Section 3. The contour will not pass through any of the singular points of $\Omega_{\widetilde{Z}}$, so the result we get will be the same as if we had integrated $\Omega_{Z}$. However, the integrand will appear to have poles that do not exist in $\Omega_{Z}$. All these poles will turn out to have zero residue.

Let us apply (3.3) to our meromorphic 3 -from in the patch $t \neq 0$ so that $t$ may be scaled to be a constant. The expression for the Kähler form becomes

$$
k=\oint_{\Gamma} \frac{d z \wedge d \zeta \wedge d x}{x(z-c)^{2}(\zeta-\bar{c})^{2}}
$$

where $\Gamma$ is a curve in a twistor line (4.15). Using $z$ to parameterize the line, we can see that $\zeta$ is given by

$$
\zeta=-\frac{A z+B}{z+\bar{A}}, \quad \text { with } A \in \mathbb{C}, B \in \mathbb{R},
$$


where without loss of generality we have scaled $H(z, \zeta)$ in (4.15) so that $b_{00}=1$. Also from (4.15), we have

$$
\frac{d x}{x}=i d \alpha+\frac{d f}{f}
$$

Note that we are using $\{\alpha, A, \bar{A}, B\}$ as coordinates on $D$. These may be held fixed as we do the $z$ integral.

The function $f$ may be factorized to give

$$
f=\prod_{i=1}^{n} f_{i}=\prod_{i=1}^{n} M_{i}\left(z-\Gamma_{i}\right)
$$

The values of $M_{i}$ and $\Gamma_{i}$ are found by requiring $f \sigma^{*}(f)=\left.P\right|_{C}$. We find

$$
\Gamma_{i}=\frac{B+2 \operatorname{Re}\left(\bar{b}_{i} A\right)-d_{i}+\sqrt{X}}{2\left(b_{i}-A\right)},
$$

where have scaled (4.7) to describe $C^{i}$ by

$$
z \zeta+b_{i} z+\bar{b}_{i} \zeta+d_{i}=0, \quad \text { with } b_{i} \in \mathbb{C}, d_{i} \in \mathbb{R},
$$

and

$$
\begin{aligned}
X= & B^{2}+d_{i}^{2}-4\left(B+d_{i}\right) \operatorname{Re}\left(\bar{b}_{i} A\right)-2 d_{i} B+2 \operatorname{Re}\left({\overline{b_{i}}}^{2} A^{2}\right)+4 d_{i}|A|^{2} \\
& +4 B\left|b_{i}\right|^{2}-2|A|^{2}\left|b_{i}\right|^{2} .
\end{aligned}
$$

In fact, $z=\Gamma_{i}$ in $C$ is a point of intersection of $C$ with $C^{i}$ because $f_{i}=0$ on $C^{i}$. The remaining coefficient in (4.41) is given as

$$
M_{i}^{2}=\frac{2|A|^{2}+d_{i}-B-2 \operatorname{Re}\left(\overline{b_{i}} A\right)+\sqrt{X}}{2\left(|A|^{2}-B\right)} .
$$

Note that $X$ is real and hence that $M_{i}^{2}$ will be real and positive if $X$ is positive. This is the case that we consider. Further note that, despite appearances to the contrary, $M_{i}$ does not have a pole at $|A|^{2}=B$ because the numerator also vanishes at this point. We may now write the second term in (4.40) as

$$
\frac{d f}{f}=\sum_{i=1}^{n} \frac{d M_{i}}{M_{i}}-\sum_{i=1}^{n} \frac{d \Gamma_{i}}{z-\Gamma_{i}} .
$$

We see that (4.46) introduces new poles into the $z$ integral in (4.38). One might worry that this would allow us to obtain different results for the Kähler form by integrating around differing contours, contradicting the setup we developed in Section 3. However, it turns out that the residues of these new poles is precisely zero, so in fact all contours around $z=c$ give the same answer. This provides a rather nice consistency check with 
the fact that in the resolved space $Z$, the 3 -form $\Omega_{Z}$ should only have poles on $D \cup \bar{D}$.

Let us perform the integration. The parts of the integral (4.38) involving $\alpha$ and $M_{i}$ give a succinct result

$$
\begin{aligned}
& \frac{1}{2 \pi i} \oint_{\Gamma} \frac{d z \wedge d \zeta}{(z-c)^{2}(\zeta-\bar{c})^{2}} \wedge\left[i d \alpha+\sum_{i=1}^{n} \frac{d M_{i}}{M_{i}}\right] \\
& \quad=-d\left[\frac{|A|^{2}-B}{\left(B+2 \operatorname{Re}(c A)+|c|^{2}\right)^{2}}\right] \wedge\left[i d \alpha+\sum_{i=1}^{n} \frac{d M_{i}}{M_{i}}\right] .
\end{aligned}
$$

Note that this contribution is manifestly closed. The explicit expression for $d M_{i} / M_{i}$ is rather large and unilluminating. The remaining terms may also be integrated

$$
\begin{aligned}
-\frac{1}{2 \pi i} & \oint_{\Gamma} \frac{d z \wedge d \zeta}{(z-c)^{2}(\zeta-\bar{c})^{2}} \wedge \sum_{i=1}^{n} \frac{d \Gamma_{i}}{z-\Gamma_{i}} \\
= & \sum_{i=1}^{n}\left(d\left[\frac{c+\bar{A}}{B+2 \operatorname{Re}(c A)+|c|^{2}}\right] \wedge \frac{d \Gamma_{i}}{\left(c-\Gamma_{i}\right)^{2}}\right. \\
& \left.-d\left[\frac{|A|^{2}-B}{\left(B+2 \operatorname{Re}(c A)+|c|^{2}\right)^{2}}\right] \wedge \frac{d \Gamma_{i}}{c-\Gamma_{i}}\right) .
\end{aligned}
$$

Once again, we see explicitly that the form is closed, as it should be. Similarly to before, the actual expression for $d \Gamma_{i}$ appears to be complicated and unhelpful. The poles in (4.47) at $M_{i}=0$ are an artifact of the coordinates we are using. From (4.41), we have that $M_{i}=0$ implies that $f=0$ and hence from (4.15) that $x=y=0$. This is a singular point on $\widetilde{Z}$ and (4.38) is not valid at this point. On the other hand, the poles in (4.48) at $c=\Gamma_{i}$ turn out to be exactly what we want. It is to these poles which we now turn.

The poles at $c=\Gamma_{i}$ for each $i=1 \cdots n$ have a geometric interpretation as follows. We noted above that $z=\Gamma_{i}$ on $C$ is the location of an intersection between $C$ and $C^{i}$. Generically, these points will not lie on the hypersurface $D$ given by $z=c$. We are using the coefficients $\{A, \bar{A}, B\}$ that define the curve $C$ as coordinates on $D$ because they are fixed for a given twistor line. The point on $D$ corresponding to a given $\{A, \bar{A}, B\}$ is the unique point where $D$ intersects $C(A, \bar{A}, B)$. Thus $c=\Gamma_{i}$ means that we are at a point on $D$ that also intersects $C^{i}$. In the discussion following (4.16), we noted that at these points the circle fibration in $D$ collapses. These $n$ points were then related to the $n$ blown-up $\mathbb{C P}^{1} \mathrm{~s}$ in $D$. The relationship between $C, C^{i}$ and $D$ is illustrated in figure 4 . 


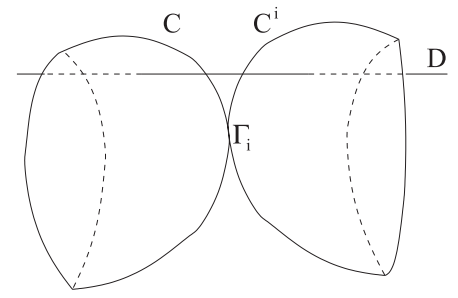

Figure 4: Generically $D=\{z=c\}$ does not go through the intersection point of $C$ and $C^{i}$. It does so when $c=\Gamma_{i}$.

To summarize the preceding paragraph: the Kähler form that we obtain has poles associated with the $n$ blown-up points of the original self-dual manifold $M$ which we started with. This is good because the explicit Kähler form for these manifolds has been written down by Lebrun [19] and has precisely this property. In principle, it should be possible to find an explicit change of variables between the 2-form we found in (4.47) and (4.48) above and the form given in [19]. However, this does not look particularly straightforward. We consider the presence of the correct poles to support the interpretation of the 3 -form (4.21) as the meromorphic 3-form corresponding to the Kähler form on $M$ via the Penrose transform.

It follows from (3.4) that when we integrate $\Omega_{Z}$ around cycles in $Z$ given by a $\mathbb{C P}^{1}$ in $D$ and a contour in the twistor line above each point of $D$, we will obtain the integral of $k$ around a 2-cycle in $M$. There are $n$ such cycles because we have blown-up $\mathbb{C}^{2}$ at $n$ points. This provides a specific realization of the map between Kähler moduli for spacetime foam and D1 brane charge.

\section{Conclusions and discussion}

We have seen that the Penrose transform provides a natural map between the Kähler moduli of Kähler anti-self-dual gravitational instantons in conformal supergravity and the D1 brane charge on the corresponding twistor spaces. This provides quantitative support for the idea that placing D1 branes in $\mathbb{C P}^{3}$ corresponds to blowing-up points in four dimensions. Such a correspondence had been anticipated by combining three ingredients: the recent conjectures of $S$-duality in topological string theory [13,14], the development of twistor string theory for $\mathbb{C P}^{3}[8]$ and the understanding of spacetime foam for the A model topological string theory on toric Calabi-Yau manifolds [4]. The hope is that this relationship may shed light upon spacetime foam in four dimensions or upon the role of D1 brane instantons in topological string theory. 
One interesting possibility that follows from the correspondence between Kähler moduli and D1 brane charge is that the quantization of the number of D1 branes should translate into a quantization of the Kähler moduli of the four dimensional quantum foam. This is a very natural expectation [4] and it would be interesting to understand any such quantization at a deeper level.

The appearance of the Kähler condition itself is also interesting. A generic anti-self-dual gravitational instanton metric is not Kähler. The correspondence suggests that the "Kähler sector" of spacetime foam is particularly amenable to study using twistor string theory. Perhaps an exact treatment of this sector is possible?

The twistor space $Z$ is not a Calabi-Yau manifold but may always be extended to a super Calabi-Yau manifold in a natural way. This extension implies the existence of a holomorphic (3|4)-form on the super Calabi-Yau. We have suggested that the meromorphic 3-form on $Z$ whose Penrose transform gives the Kähler form could be obtained by integrating the (3|4)-form over the fermionic directions. We showed explicitly how this could work for the twistor spaces corresponding to blowups of $\mathbb{C}^{2}$. In this picture, the D1 brane charge is detected by integrating the holomorphic (3|4)-form over a (3|4)-cycle in the super Calabi-Yau. This notion does not depend on the Kähler structure of the four dimensional manifold. By using the superforms, it should be possible to extend the correspondence between spacetime foam and D1 brane charge to general anti-self-dual gravitational instantons.

An alternative construction of the twistor spaces for blowups that does not emphasize the Kähler property is [16]. In that work, the connected sum of an anti-self-dual manifold $M$ and, say, $\overline{\mathbb{C P}^{2}}$ was formed as follows. One performs a real blowup of a point in $M$ and a point in $\overline{\mathbb{C P}^{2}}$. A real blowup replaces a point with an $\mathbb{R P}^{3}$. One then glues the two copies of $\mathbb{R P}^{3}$ together to obtain the connected sum. In twistor space, the real blowup corresponds to a complex blowup along the $\mathbb{C P}^{1} \mathrm{~s}$ corresponding to the two points. The two exceptional divisors are then glued together and under certain conditions the resulting singular space may be smoothed to give a new twistor space. In this case, the corresponding four dimensional manifold $M \# \overline{\mathbb{C P}^{2}}$ is again anti-self-dual. Realizing this construction within topological string theory could be one way to extend the correspondence we have been studying to general blowups.

Finally, it would be interesting to understand the four dimensional interpretation of the $\mathrm{D}(-1)$ branes in the topological $\mathrm{B}$ model on twistor space [13]. 


\section{Acknowledgments}

We are very grateful to Ivan Smith and Maciej Dunajski for patient and helpful explanations of various aspects of algebraic geometry and twistor theory, respectively. We would also like to thank Robbert Dijkgraaf, Prem Kumar, Fabien Morel and Peter Mayr for useful discussions. S.A.H. would like to thank the second Simons Workshop at SUNY Stony Brook for providing a stimulating environment combining both twistors and topological strings. S.A.H. is supported by a research fellowship from Clare College, Cambridge. G.P. is supported by the SFB 375 of DFG.

\section{Appendix A Jet bundles}

In this appendix, we give some technical details on the geometry of 1-jet bundles, $J^{1}(L)$ [33]. Let $L$ be a line bundle over a complex manifold $X$. There is an exact sequence

$$
0 \longrightarrow \Omega^{1} \otimes L \stackrel{j}{\longrightarrow} J^{1}(L) \stackrel{p}{\longrightarrow} L \longrightarrow 0 .
$$

This tells us that $J^{1}(L)$ is an extension of $L$. Locally the sequence is split, which means that given a cover of $X$ by $U_{i}$ such that all the bundles involved are trivial on the $U_{i}$ and their intersections, there are maps $h_{i}: L_{i} \rightarrow J^{1}\left(L_{i}\right)$ such that $p \circ h_{i}=I d$. Together with the injection $j$, this gives local isomorphisms $u_{i}^{-1}:\left.\left(\left[\Omega^{1} \otimes L\right] \oplus L\right)\right|_{i} \rightarrow J^{1}\left(L_{i}\right),(a, b) \mapsto j(a)+h_{i}(b)$. The inverse map is $u_{i}(s)=\left(j^{-1}\left(s-h_{i} \circ p(s)\right), p(s)\right)$. Then

$$
u_{i} \circ u_{j}^{-1}(a, b)=\left(a+j^{-1}\left(h_{j}(b)-h_{i}(b)\right), b\right) .
$$

This shows that the class of the extension, which can be thought of as the obstruction to a global splitting of the sequence (A.1), is defined by a 1-cocycle $\left\{h_{i j}\right\}, h_{i j}=j^{-1}\left(h_{j}-h_{i}\right)$, with values in $\operatorname{Hom}\left(L, \Omega^{1} \otimes L\right) \cong \Omega^{1}$. This cocycle is intrinsically defined as follows: tensoring the sequence (A.1) with $L^{*}$, one obtains

$$
0 \longrightarrow \Omega^{1} \longrightarrow J^{1}(L) \otimes L^{*} \longrightarrow \underline{\mathbb{C}} \longrightarrow 0,
$$

where $\mathbb{C}$ is the trivial line bundle. Associated to this sequence, there is a long exact cohomology sequence with, in particular, a connecting map $\delta: H^{0}(\underline{\mathbb{C}}) \rightarrow H^{1}\left(\Omega^{1}\right)$. The trivial line bundle has a global section which is just the constant function 1 ; its image under $\delta$ is the class of the extension. In local coordinates, if $g_{i j}$ are the transition functions of $L$, it may be shown that [33]

$$
h_{i j}=d \ln g_{i j},
$$

and therefore the extension class is just $c_{1}(L)$. In particular, we see that in the case of twistor spaces, where we take $L$ to be $K^{-1 / 2}$, the first Chern 
class does not vanish and therefore the sequence is not split, as claimed in the text.

We can now give an explicit description of $J^{1}(L)$ in terms of its transition functions $G_{i j}$. Given local trivializations $\phi_{i}: L_{i} \rightarrow U_{i} \times \mathbb{C}$, and $\psi_{i}$ : $\Omega_{i}^{1} \rightarrow U_{i} \times \mathbb{C}^{3}$, one has $\Phi_{i}=\left(\left[\psi_{i} \otimes \phi_{i}\right] \oplus \phi_{i}\right) \circ u_{i}: J^{1}\left(L_{i}\right) \rightarrow U_{i} \times\left(\mathbb{C}^{3} \oplus \mathbb{C}\right)$ as trivializations for $J^{1}(L)$. Then

$$
G_{i j}=\Phi_{i} \Phi_{j}^{-1}:(v, s) \longmapsto\left(g_{i j}^{\Omega} g_{i j}^{L}(v)+\left(\psi_{i} h_{i j}\right) g_{i j}^{L}(s), g_{i j}^{L}(s)\right),
$$

where $g_{i j}^{L}$ and $g_{i j}^{\Omega}$ denote the transition functions for $L$ and $\Omega^{1}$, respectively.

It is useful to observe that $J^{1}\left(L^{n}\right) \cong J^{1}(L) \otimes L^{n-1}$. This can be seen by comparing two exact sequences: one obtained from (A.1) by taking $L^{n}$ as the line bundle and the other by tensoring (A.1) with $L^{n-1}$.

The case we are interested in is $L=K_{Z}^{-1 / 2}$ for a twistor space $Z$. We would like to compute the restriction of $J^{1}(L)$ to a twistor line $C \cong \mathbb{C P}^{1}$. First, using the adjunction formula, one can see that $\left.K\right|_{C} \cong \mathcal{O}(-4)$, so $\left.L\right|_{C} \cong \mathcal{O}(2)$. The observation made before gives $J^{1}(\mathcal{O}(2)) \cong J^{1}(\mathcal{O}(1)) \otimes$ $\mathcal{O}(1)$. We also need the fact that $\left.\Omega_{Z}^{1}\right|_{C} \cong \Omega_{C}^{1} \oplus N_{C \mid Z}^{*} \cong \mathcal{O}(-2) \oplus \mathcal{O}(-1) \oplus$ $\mathcal{O}(-1)$. Restricting the exact sequence (A.1) to $C$, one obtains

$$
0 \longrightarrow \mathcal{O}(-1) \oplus \mathcal{O} \oplus \mathcal{O} \longrightarrow J^{1}(\mathcal{O}(1)) \longrightarrow \mathcal{O}(1) \longrightarrow 0
$$

The transition function for $\mathcal{O}(1)$ is $g_{i j}=z$. Using (A.5) and noting that the extension of (A.6) only involves the $\mathcal{O}(-1)$ summand in the first term - corresponding to 1 -forms on $C$, the other summands correspond to the normal bundle of $C$ - we can write the nontrivial part of the transition matrix for $J^{1}(\mathcal{O}(1))$ as

$$
G_{i j}=\left(\begin{array}{cc}
\frac{1}{z} & \frac{1}{z^{2}} \\
0 & z
\end{array}\right)
$$

corresponding to a rank 2 vector bundle $E$ over $C \cong \mathbb{P}^{1}$. As is well known, every such bundle is isomorphic to a direct sum $E=\mathcal{O}\left(k_{1}\right) \oplus \mathcal{O}\left(k_{2}\right)$. From (A.7), we have that $k_{1}+k_{2}=c_{1}(E)=c_{1}(\operatorname{det} E)=1-1=0$. Now consider $E \otimes \mathcal{O}(-1) \cong \mathcal{O}\left(k_{1}-1\right) \oplus \mathcal{O}\left(k_{2}-1\right)$, which has transition matrix $G_{i j} z^{-1}$. If this bundle has a global section, given in the two charts of $\mathbb{C P}^{1}$ by vectors $\left(a_{1}(z), a_{2}(z)\right)$ and $\left(b_{1}(\tilde{z}), b_{2}(\tilde{z})\right)$, one has

$$
\left(\begin{array}{l}
a_{1}(z) \\
a_{2}(z)
\end{array}\right)=\left(\begin{array}{cc}
\frac{1}{z^{2}} & \frac{1}{z^{3}} \\
0 & 1
\end{array}\right)\left(\begin{array}{l}
b_{1}(\tilde{z}) \\
b_{2}(\tilde{z})
\end{array}\right) .
$$

Matching the power series in $z, \tilde{z}$, one can see the only solution is $b_{1}=b_{2}=0$. Therefore, $E \otimes \mathcal{O}(-1)$ has no global sections, which implies that $k_{1}, k_{2}<1$, 
and therefore $k_{1}=k_{2}=0$. Thus, we have that $J^{1}(\mathcal{O}(1))=\oplus_{i=1}^{4} \mathcal{O}$ which gives the result stated in the text

$$
\left.J^{1}(L)\right|_{C}=\bigoplus_{i=1}^{4} \mathcal{O}(1) .
$$

\section{Appendix B Calculation of $b_{3}(\widetilde{Z})$}

In this appendix, we compute the first Betti number of $X^{n+1}=\cup_{i=1}^{n+1} C^{i}$, where the $C^{i}$ s are 2-cycles that all mutually intersect at two points. An illustration was given in figure 1 in the text.

Without changing the Betti numbers, we may homotopically stretch the intersection points so that they become discs: $C^{i} \cap C^{j}=D^{2} \sqcup D^{2}$. We may now calculate easily using part of the Mayer-Vietoris sequence

$$
\begin{aligned}
\cdots & \longrightarrow H_{1}\left(X_{1} \cap X_{2}\right) \longrightarrow H_{1}\left(X_{1}\right) \oplus H_{1}\left(X_{2}\right) \rightarrow H_{1}(X) \longrightarrow \\
& \longrightarrow H_{0}\left(X_{1} \cap X_{2}\right) \longrightarrow H_{0}\left(X_{1}\right) \oplus H_{0}\left(X_{2}\right) \longrightarrow H_{0}(X) \longrightarrow 0 .
\end{aligned}
$$

In particular, we take $X_{1}=\cup_{i=1}^{n} C^{i}$ and $X_{2}=C^{n+1}$. The sequence (B.1) becomes

$$
0 \longrightarrow H_{1}\left(X^{n}\right) \oplus 0 \longrightarrow H_{1}\left(X^{n+1}\right) \longrightarrow \oplus_{j=1}^{2 n} \mathbb{Z} \longrightarrow \mathbb{Z} \oplus \mathbb{Z} \longrightarrow \mathbb{Z} \longrightarrow 0 .
$$

The exactness of this sequence then implies that

$$
H_{1}\left(X^{n+1}\right)=H_{1}\left(X^{n}\right) \bigoplus_{j=1}^{2 n-1} \mathbb{Z} .
$$

Taking $b_{1}=\operatorname{dim} H_{1}$ of (B.3) and using induction together with the fact that $b_{1}\left(X^{1}\right)=0$, one obtains

$$
b_{1}\left(X^{n}\right)=b_{3}(\widetilde{Z})=(n-1)^{2} .
$$

\section{Appendix C Geometric transitions and topology of the twistor space}

This appendix calculates directly the Betti numbers of the twistor space $Z$. In the course of the calculation, we shall also consider deformations of the singular space $\widetilde{Z}$ that are related to the twistor space by geometric transitions. 
The logic we follow is to start with a singular projective variety $\widetilde{Z}$. The singularities are given by nodal points. We will deform this space to a nonsingular variety $\widetilde{Z}_{\text {def. }}$. It is straightforward to calculate the Betti numbers of this deformed variety and then perform geometric transitions at the deformed singular points to obtain a small resolution of the initial singular space $\widetilde{Z}_{\text {res. }}$. The advantage of doing this is that we can now read off the Betti numbers of the resolved space, which would have been harder to calculate directly. Finally, the twistor space $Z$ itself is given by blowing down two divisors in the resolved space. Schematically:

$$
\widetilde{Z} \stackrel{\text { deform }}{\longrightarrow} \widetilde{Z}_{\text {def. }} \stackrel{\text { transition }}{\longrightarrow} \widetilde{Z}_{\text {res. }} \stackrel{\text { blowdown }}{\longrightarrow} Z \text {. }
$$

The deformed space is given by

$$
x y=t^{2}\left[\prod_{j=1}^{n} P^{j}+\epsilon P^{n, n}\right],
$$

where $\epsilon$ is a small parameter and $P^{n, n}$ is a generic polynomial with homogeneity $n$ in $z_{m}$ and $\zeta_{m}$. The right hand side of the equation for the deformed space (C.2) will not have double roots and the resulting space will be regular.

The deformation will replace the singular points with finite $S^{3} \mathrm{~s}$. The strategy is now as follows. We will first calculate $b_{3}\left(\widetilde{Z}_{\text {def }}\right)$ and $b_{2}\left(\widetilde{Z}_{\text {def }}\right)$. The geometric transition then degenerates these cycles and replaces them with the $\mathbb{C P}^{1} s$ that are generated by the small resolution. There is a relationship between the Betti numbers before and after the geometric transition [34, Theorem 2.11],

$$
\begin{aligned}
& b_{3}\left(\widetilde{Z}_{\text {res. }}\right)=b_{3}\left(\widetilde{Z}_{\text {def. }}\right)-2 r, \\
& b_{2}\left(\widetilde{Z}_{\text {res. }}\right)=b_{2}\left(\widetilde{Z}_{\text {def. }}\right)+n(n-1)-r,
\end{aligned}
$$

where $n(n-1)$ is the number of nodes of the singular space and where the degenerating 3-cycles span an $r$ dimensional subset of $H_{3}\left(\widetilde{Z}_{\text {def. }}\right)$. More precisely

$$
r=b_{3}\left(\widetilde{Z}_{\text {def. }}\right)-b_{3}(\widetilde{Z})
$$

The result (C.3) is intuitively reasonable [34]. For the first line in (C.3), we can think that for every 3 -cycle we degenerate, we lose another one by Poincaré duality. For the second line, we should think about how the $r$ 3 -cycles that degenerate give homology relations between the new 2-cycles. A 2-cycle is created at each of the $n(n-1)$ nodes. However, these can be the boundaries of three-chains that were 3 -cycles before the transition. 
Note that the formulae in (C.3) do not apply to the well-known conifold transition because in that case the manifolds involved are noncompact and Poincaré duality is different.

We can get $b_{3}\left(\widetilde{Z}_{\text {def. }}\right)$ using the same method as in the singular case. The same arguments as in Appendix B imply that

$$
b_{3}\left(\widetilde{Z}_{\text {def. }}\right)=b_{1}\left(X_{\text {def. }}^{n}\right)
$$

where $X_{\text {def. }}^{n}$ is the zero space of the deformed polynomial in (C.2). The deformation has smoothed out the points of intersection, so $X_{\text {def. }}^{n}$ is now just a Riemann surface. This is illustrated in figure C.1. We can calculate $b_{1}\left(X_{\text {def. }}^{n}\right)$ in a similar fashion to before, using the Mayer-Vietoris sequence or by applying the adjunction formula to the degree $(n, n)$ polynomial. Alternatively, we can easily see directly that $X_{\text {def. }}^{n}$ is a Riemann surface with genus $(n-1)^{2}$. Therefore, we have

$$
b_{3}\left(\widetilde{Z}_{\text {def. }}\right)=b_{1}\left(X_{\text {def. }}^{n}\right)=2(n-1)^{2} .
$$

\section{C.1 Calculation of $b_{2}\left(\widetilde{Z}_{\text {def. }}\right)$}

In order to get $b_{2}\left(\widetilde{Z}_{\text {def. }}\right)$, we calculate the Euler character of the deformed space.

Recall the following two properties of the Euler character

$$
\begin{aligned}
\chi(A \cup B) & =\chi(A)+\chi(B)-\chi(A \cap B), \\
\chi(A \times B) & =\chi(A) \chi(B) .
\end{aligned}
$$

We will write

$$
\widetilde{Z}_{\text {def. }}=A \cup B,
$$

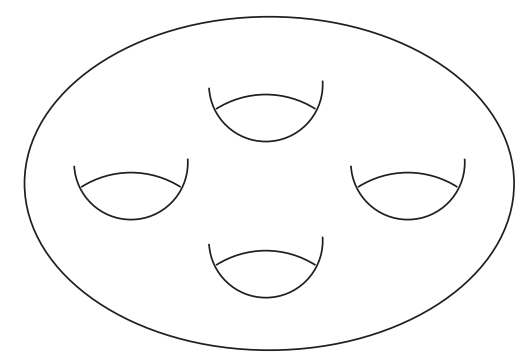

Figure C.1: The Riemann surface resulting from smoothing the intersections in figure 1. 
with $A$ being the degenerate fibration over $X_{\text {def. }}^{n}$

$$
A=X_{\text {def. }}^{n} \times\left(S^{2} \vee S^{2}\right),
$$

and $B$ the nondegenerate $\mathbb{C P}^{1}$ fibration over the remainder of $\mathbb{C P}^{1} \times \mathbb{C P}^{1}$, see figure 1,

$$
B=\widetilde{Z}_{\text {def. }} \backslash\left[X_{\text {def. }}^{n} \times\left(S^{2} \vee S^{2}\right)\right]=\mathbb{C P}^{1} \times\left[\mathbb{C P}^{1} \times \mathbb{C P}^{1} \backslash X_{\text {def. }}^{n}\right],
$$

where again $S^{2} \vee S^{2}$ denotes two spheres joined at a point.

Therefore, we have

$$
\chi(A)=3 \chi\left(X_{\text {def. }}^{n}\right)
$$

and

$$
\chi(B)=2 \chi\left(\mathbb{C P}^{1} \times \mathbb{C P}^{1} \backslash X_{\text {def. }}^{n}\right)=2\left[4-\chi\left(X_{\text {def. }}^{n}\right)\right] .
$$

Putting these two results together and using the fact that $A \cap B=\emptyset$, we have that

$$
\chi\left(\widetilde{Z}_{\text {def. }}\right)=8+\chi\left(X_{\text {def. }}^{n}\right)=10-b_{1}\left(X_{\text {def. }}^{n}\right) .
$$

First, note that

$$
b_{1}(Z)=b_{1}\left(\widetilde{Z}_{\text {def. }}\right)=b_{1}\left(\widetilde{Z}_{\text {res. }}\right)=0,
$$

and similarly for $b_{5}$ by Poincaré duality. Note that because all the relevant spaces are now nonsingular, we may apply Poincaré duality without complications. The first Betti number of the twistor space $Z$ vanishes because $Z$ is a $\mathbb{C P}^{1}$ bundle over a simply connected four-manifold $M$. It therefore follows from Leray's theorem $[24]$ that $b_{1}(Z)=0$. The other two spaces are related to $Z$ by blowups, blowdowns and geometric transitions which do not change the first Betti number.

Finally, using the fact that $b_{3}(\widetilde{Z})=b_{1}\left(X^{n}\right)$ and Poincaré duality, it follows from (C.13) that

$$
b_{2}\left(\widetilde{Z}_{\text {def. }}\right)=4
$$

\section{C.2 Betti numbers of the resolved space}

Now we can calculate $b_{3}\left(\widetilde{Z}_{\text {res. }}\right)$ and $b_{2}\left(\widetilde{Z}_{\text {res. }}\right)$ from (C.3) and (C.4) to find

$$
b_{3}\left(\widetilde{Z}_{\text {res. }}\right)=2(n-1)^{2}-2(n-1)^{2}=0 \text {, }
$$

and

$$
b_{2}\left(\widetilde{Z}_{\text {res. }}\right)=4+n(n-1)-(n-1)^{2}=n+3 .
$$

The last step is to go from $\widetilde{Z}_{\text {res. }}$ to $Z$ by performing two blowdowns. The two surfaces $x=t=0$ and $y=t=0$ in $\widetilde{Z}$ give two copies of $\mathbb{C P}^{1} \times \mathbb{C P}^{1}$. These may be blown down [19] to give two $\mathbb{C P}^{1} \mathrm{~s}$ as we described in the 
text. The only effect of this blowdown is to reduce the second Betti number by two

$$
b_{2}(Z)=b_{2}\left(\widetilde{Z}_{\text {res. }}\right)-2 \text {. }
$$

Therefore, we obtain

$$
b_{3}(Z)=0, \quad b_{2}(Z)=n+1 .
$$

It is immediately seen that this result is consistent with the cases $n=0$ and $n=1$, where the twistor spaces are $\mathbb{C P}^{3}$ and the flag manifold $F_{3}(\mathbb{C})$, respectively. It is also consistent with the general result given in Section 2.1.

\section{Appendix D Small resolution versus blowup}

In this appendix, we show how a small resolution can be obtained by means of blowups. Since all the considerations are local, we consider, as in the text, the singular conifold in $\mathbb{C}^{4}$

$$
V=\left\{z_{1} z_{2}-z_{3} z_{4}=0\right\} .
$$

The small resolution is, as we have seen, the total space of a bundle

$$
X=\mathcal{O}(-1)[u] \oplus \mathcal{O}(-1)[v] \longrightarrow \mathbb{C P}^{1}\left[Z_{1}, Z_{2}\right],
$$

where we have put in square brackets the coordinates for the respective spaces.

First, we want to show that the blowup of $X$ along the $\mathbb{C P}^{1}$ given by the zero section $M=\{u=v=0\}$ can be identified with the total space of a line bundle:

$$
\tilde{X}=\mathcal{O}(-1,-1)[y] \longrightarrow \mathbb{C P}^{1}\left[Y_{1}, Y_{2}\right] \times \mathbb{C P}^{1}\left[Z_{1}, Z_{2}\right] .
$$

We give the blowup map $\pi: \tilde{X} \rightarrow X$ as follows:

$$
\begin{aligned}
& \left\{Y_{1} \neq 0\right\}, \quad\left\{u=y^{(1)}, v=\frac{Y_{2}}{Y_{1}} y^{(1)}, Z=Z\right\} \\
& \left\{Y_{2} \neq 0\right\}, \quad\left\{u=\frac{Y_{1}}{Y_{2}} y^{(2)}, v=y^{(2)}, Z=Z\right\} .
\end{aligned}
$$

One can see that the map is well defined, is invertible if $u \neq 0$ or $v \neq 0$, and the inverse image of a point on $M$ is a $\mathbb{C P}^{1}$ parametrized by $Y_{1}, Y_{2}$. This is enough to prove that $\tilde{X}$ is the blowup of $X$ along $M$.

Secondly, we show that $\tilde{X}$ can also be interpreted as the proper transform of the singular conifold $V$ under the blowup of $\mathbb{C}^{4}$ at the origin. The latter is defined as

$$
\tilde{\mathbb{C}}^{4}=\left\{\left(z_{i}\right) \in \mathbb{C}^{4},\left[l_{i}\right] \in \mathbb{C P}^{3} \mid z_{i} l_{j}=z_{j} l_{i}\right\}
$$


We cover the blowup with charts $U_{i}=\left\{l_{i} \neq 0\right\}$ and use local coordinates $x^{(i)}$ defined by

$$
x_{i}^{(i)}=z_{i}, \quad x_{j}^{(i)}=\frac{l_{j}}{l_{i}}, j \neq i .
$$

The exceptional divisor $E \simeq \mathbb{C P}^{3}$ is given in $U_{i}$ by $\left\{x_{i}^{(i)}=0\right\}$. The proper transform of $V$ is now obtained by looking at its equation in local coordinates:

$$
\begin{aligned}
& U_{1}: x_{2}^{(1)}=x_{3}^{(1)} x_{4}^{(1)}, \\
& U_{2}: x_{1}^{(2)}=x_{3}^{(2)} x_{4}^{(2)}, \\
& U_{3}: x_{4}^{(3)}=x_{1}^{(3)} x_{2}^{(3)}, \\
& U_{4}: x_{3}^{(4)}=x_{1}^{(4)} x_{2}^{(4)} .
\end{aligned}
$$

One can check that $\tilde{V} \cap E=\left\{z_{i}=0, l_{1} l_{2}-l_{3} l_{4}=0\right\}$, which exhibits the $\mathbb{C P}^{1} \times \mathbb{C P}^{1}$ (take $\left.l_{1}=Z_{1} Y_{1}, l_{2}=Z_{2} Y_{2}, l_{3}=Z_{1} Y_{2}, l_{4}=Z_{2} Y_{1}\right)$. The remaining coordinate parametrizing $\tilde{V}$ is $x_{i}^{(i)}$, and using the equations (D.5) one can see that it has the right transformation properties to be a coordinate on the fibre of the $\mathcal{O}(-1,-1)$ bundle. Thus $\tilde{V}=\tilde{X}$, and we see that we have two routes from $V$ to the small resolution $X$ :

$$
V \stackrel{\text { small res. }}{\longleftrightarrow} X \stackrel{\text { blowdown }}{\longleftarrow} \tilde{X}=\tilde{V} \stackrel{\text { proper xfm. }}{\longleftarrow} V \text {. }
$$

Starting from $\tilde{X}$, one can also obtain a different space $X^{\prime}$, isomorphic to $X$, by blowing down the other $\mathbb{C P}^{1}$, parametrized by $Z$. The transition between $X$ and $X^{\prime}$ is called a flop.

Notice that if, in the context of Section 4 in the text, one sees the conifold as a fibration over the $z_{3}, z_{4}$ plane which degenerates at $C^{1}=\left\{z_{3}=0\right\}$ and $C^{2}=\left\{z_{4}=0\right\}$, taking the proper transforms of the $C^{i}$, one finds that

$$
\begin{aligned}
& \tilde{C}^{1} \cap E=\left\{\left[l_{i}\right]=[0,0,0,1]\right\}=\{[Z]=[0,1],[Y]=[1,0]\} \\
& \tilde{C}^{2} \cap E=\left\{\left[l_{i}\right]=[0,0,1,0]\right\}=\{[Z]=[1,0],[Y]=[0,1]\} .
\end{aligned}
$$

Therefore, the two curves remain disjoint in the resolution, regardless of which of the two $\mathbb{C P}^{1}$ is blown down.

\section{References}

[1] S.W. Hawking, Space-time foam, Nucl. Phys. B 144 (1978), 349.

[2] J.A. Wheeler, in Relativity groups and topology, eds. B.S. DeWitt and C.M. DeWitt, Gordon and Breach, New York, 1964. 
[3] M. Bershadsky and V. Sadov, Theory of Kahler gravity, Int. J. Mod. Phys. A 11 (1996), 4689, arXiv:hep-th/9410011.

[4] A. Iqbal, N. Nekrasov, A. Okounkov and C. Vafa, Quantum foam and topological strings, arXiv:hep-th/0312022.

[5] E. Witten, Two-dimensional gravity and intersection theory on moduli space, Surveys Diff. Geom. 1 (1991), 243.

[6] M. Aganagic, A. Klemm, M. Marino and C. Vafa, The topological vertex, arXiv:hep-th/0305132.

[7] A. Okounkov, N. Reshetikhin and C. Vafa, Quantum Calabi-Yau and classical crystals, arXiv:hep-th/0309208.

[8] E. Witten, Perturbative gauge theory as a string theory in twistor space, arXiv:hep-th/0312171.

[9] N. Berkovits and E. Witten, Conformal supergravity in twistor-string theory, arXiv:hep-th/0406051.

[10] R. Penrose, Nonlinear gravitons and curved twistor theory, Gen. Rel. Grav. 7 (1976), 31.

[11] M.F. Atiyah, N.J. Hitchin and I.M. Singer, Selfduality in fourdimensional Riemannian geometry, Proc. Roy. Soc. Lond. A 362 (1978), 425.

[12] E.S. Fradkin and A.A. Tseytlin, Conformal supergravity, Phys. Rept. 119 (1985), 233.

[13] N. Nekrasov, H. Ooguri and C. Vafa, S-duality and topological strings, JHEP 0410:009 (2004); arXiv:hep-th/0403167.

[14] A. Neitzke and C. Vafa, $N=2$ strings and the twistorial Calabi-Yau, arXiv:hep-th/0402128.

[15] C. Taubes, The existence of anti-self-dual metrics, J. Differential Geom. 36 (1992), 163.

[16] S. Donaldson and R. Friedman, Connected sums of self-dual manifolds and deformations of singular spaces, Nonlinearity 2 (1989), 197.

[17] C. Lebrun, Anti-self-dual metrics and Kähler geometry, in Proceedings of the International Congress of Mathematicians (Zürich, 1994), Birkhäuser, Basel, 1995, pp. 498-507.

[18] C. Lebrun, Anti-self-dual Riemannian 4-manifolds, in Twistor theory (Plymouth), 81-94, Lect. Notes in Pure and Applied Mathematics, vol. 169, Dekker, New York, 1995.

[19] C. Lebrun, Explicit self-dual metrics on $\mathbb{C P} \# \cdots \# \mathbb{C P}$, J. Differential Geom. 34 (1991), 223.

[20] C. Lebrun, Twistors, Kahler manifolds and bimeromorphic geometry. I, J. Am. Math. Soc., 5 (1992), 289. 
[21] F. Campana, On twistor spaces of the class $\mathcal{C}$, J. Differential geometry 33 (1991), 541.

[22] A. Strominger, Vacuum topology and incoherence in quantum gravity, Phys. Rev. Lett. 52 (1984), 1733.

[23] A. Strominger, G.T. Horowitz and M.J. Perry, Instantons in conformal gravity, Nucl. Phys. B 238 (1984), 653.

[24] R. Bott and L.W. Tu, Differential forms in algebraic topology, SpringerVerlag, New York, 1982.

[25] J.W. Milnor and J.D. Stasheff, Characteristic classes, Princeton University Press, Princeton, New Jersey, 1974.

[26] C. Lebrun, Talk given at the second Simons workshop at Stony Brook, available at http://insti.physics.sunysb.edu/itp/conf/simonsworkII.

[27] N.J. Hitchin, Kählerian twistor spaces, Proc. London Math. Soc. 43 (1981), 133.

[28] M. Pontecorvo, On twistor spaces of anti-self-dual Hermitian surfaces, Trans. Am. Math. Soc. 331 (1992), 653.

[29] M. Aganagic, R. Dijkgraaf, A. Klemm, M. Marino and C. Vafa, Topological strings and integrable hierarchies, arXiv:hep-th/0312085.

[30] Y.S. Poon, On the algebraic structure of twistor spaces, J. Differential Geom. 36 (1992), 451.

[31] P. Griffiths and J. Harris, Principles of algebraic geometry, Wiley, New York, 1994.

[32] S. Sethi, Supermanifolds, rigid manifolds and mirror symmetry, Nucl. Phys. B 430 (1994), 31; arXiv:hep-th/9404186.

[33] M.F. Atiyah, Complex analytic connections in fibre bundles, Trans. Amer. Math. Soc. 85 (1957), 181-207.

[34] I. Smith, R.P. Thomas and S.-T. Yau, Symplectic conifold transitions, J. Differential Geom. 62 (2002), 209. 